\title{
Low-frequency RF Coupling To Unconventional (Fat Unbalanced) Dipoles
}

Mike M. Ong, Charles G. Brown Jr., Michael P. Perkins, Ronnie D. Speer, and Jalal (Jay) B. Javedani 


\section{Auspice}

This work performed under the auspices of the U.S. Department of Energy by Lawrence Livermore National Laboratory under Contract DE-AC52-07NA27344.

\section{Disclaimer}

This document was prepared as an account of work sponsored by an agency of the United States government. Neither the United States government nor Lawrence Livermore National Security, LLC, nor any of their employees makes any warranty, expressed or implied, or assumes any legal liability or responsibility for the accuracy, completeness, or usefulness of any information, apparatus, product, or process disclosed, or represents that its use would not infringe privately owned rights. Reference herein to any specific commercial product, process, or service by trade name, trademark, manufacturer, or otherwise does not necessarily constitute or imply its endorsement, recommendation, or favoring by the United States government or Lawrence Livermore National Security, LLC. The views and opinions of authors expressed herein do not necessarily state or reflect those of the United States government or Lawrence Livermore National Security, LLC, and shall not be used for advertising or product endorsement purposes. 


\section{Table of Contents}

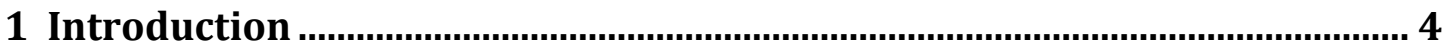

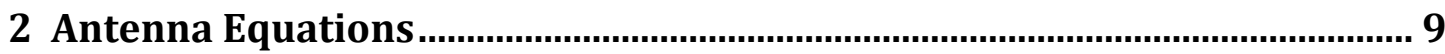

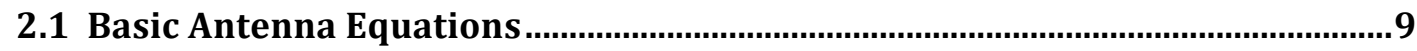

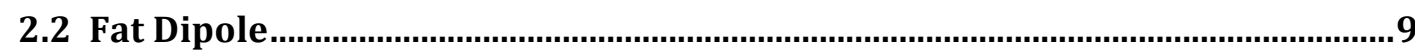

2.3 Antenna Loading - Capacitive Divider......................................................... 10

3 Computer Modeling ....................................................................................13

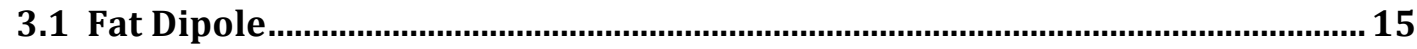

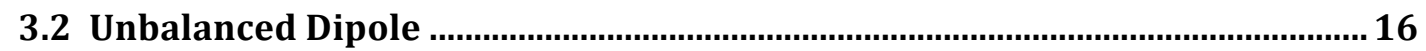

3.3 Gap Spacing and Loading ........................................................................... 19

3.4 Antenna-to-Ground Separation.................................................................. 20

3.5 Circuit Modeling of Antenna Loading ............................................................. 22

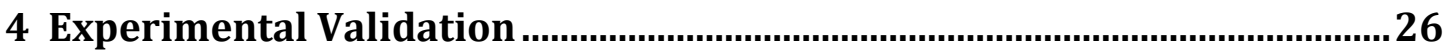

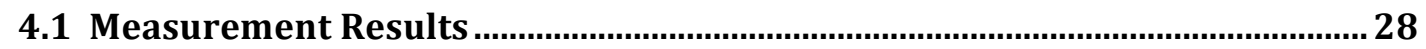

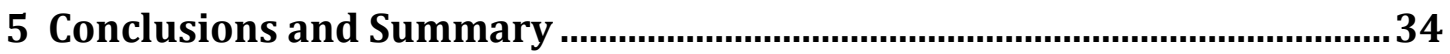

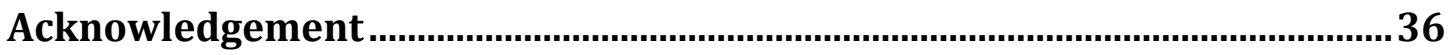

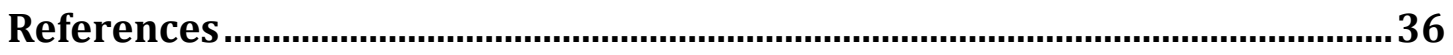

Appendix A - High Resolution Models Of Unbalanced Dipoles - Javedani.... 38

Method 1: Zoom Technique in 2D MAXWELL ........................................................38

Method 2: Dummy Geometry Technique in 2D.....................................................40

Method 3: Dummy Geometry Technique in 3D ........................................................41 


\section{Introduction}

The report explains radio frequency (RF) coupling to unconventional dipole antennas. Normal dipoles have thin equal length arms that operate at maximum efficiency around resonance frequencies. In some applications like high-explosive (HE) safety analysis, structures similar to dipoles with "fat" unequal length arms must be evaluated for indirect-lightning effects. An example is shown in Figure 1.1 where a metal drumshaped container with HE forms one arm and the detonator cable acts as the other. Even if the $\mathrm{HE}$ is in a facility converted into a "Faraday cage", a lightning strike to the facility could still produce electric fields inside [1.1 - Clancy]. The detonator cable concentrates the electric field and carries the energy into the detonator, potentially creating a hazard. This electromagnetic (EM) field coupling of lightning energy is the indirect effect of a lightning strike.

In practice, "Faraday cages" are formed by the rebar of the

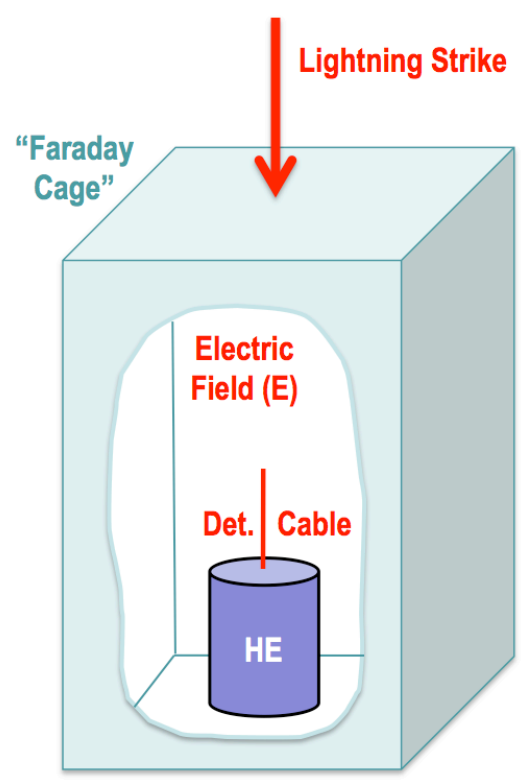

Figure 1.1. A lightning strike will create electric fields in a "Faraday cage". concrete facilities. The individual rebar rods in the roof, walls and floor are normally electrically connected because of the construction technique of using metal wire to tie the pieces together. There are two additional requirements for a good cage. (1) The roof-wall joint and the wall-floor joint must be electrically attached. (2) All metallic penetrations into the facility must also be electrically connected to the rebar. In this report, it is assumed that these conditions have been met, and there is no arcing in the facility structure.

Many types of detonators have metal "cups" that contain the explosives and thin electrical initiating wires, called bridge wires mounted between two pins. The pins are connected to the detonator cable. The area of concern is between the pins supporting the bridge wire and the metal cup forming the outside of the detonator. Detonator cables usually have two wires, and in this example, both wires generated the same voltage at the detonator bridge wire. This is called the commonmode voltage. (See Figure 1.2.) The explosive component inside a detonator is relatively sensitive, and any electrical arc is a concern. In a safety analysis, the pin-to-cup voltage, i.e., detonator voltage, must be calculated to decide if an arc will form. If the electric field is known, the voltage between any two points is simply the integral of the field along a line between the points. Eq. 1.1. For simplicity, it is assumed that the electric field and dipole elements are aligned. Calculating the induced detonator voltage is more complex because of the field concentration caused by metal components, Eq. 1.2.

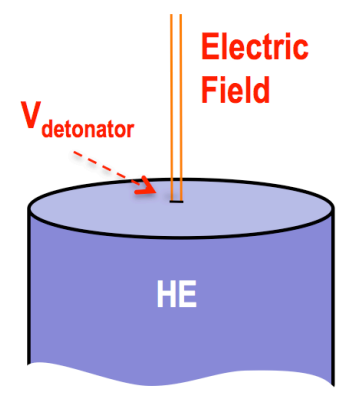

Figure 1.2. The detonator voltage can be determined by the integral of the electrical field in the gap. 


$$
\begin{aligned}
& V(t)=-\int \vec{E}(t) \cdot \mathrm{d} \overrightarrow{\mathrm{I}} \\
& V(t)_{\text {detonator }}=-\int_{\text {pin-to-cup }} \vec{E}(t) \cdot \mathrm{d} \overrightarrow{\mathrm{I}}
\end{aligned}
$$

If the detonator cup is not electrically connected to the metal HE container, the portion of the voltage generated by the dipole at the detonator will divide between the container-to-cup and cup-to-pin gaps. The gap voltages are determined by their capacitances. As a simplification, it will be assumed the cup is electrically attached, short circuited, to the HE container.

The electrical field in the pin-to-cup area is determined by the field near the dipole, the length of the dipole, the shape of the arms, and the orientation of the arms. Given the characteristics of a lightning strike and the inductance of the facility, the electric fields in the "Faraday cage" can be calculated. The important parameters for determining the voltage in an empty facility are the inductance of the rebars and the rate of change of the current, Eq. 1.3. The internal electric fields are directly related to the facility voltages, however, the electric fields in the pin-to-cup space is much higher than the facility fields because the antenna will concentrate the fields covered by the arms. Because the lightning current rise-time is different for every strike, the maximum electric field and the induced detonator voltage should be described by probability distributions. For pedantic purposes, the peak field in the simulations will be simply set to $1 \mathrm{~V} / \mathrm{m}$. Lightning induced detonator voltages can be calculated by scaling up with the facility fields.

$$
V(t)=L_{\text {Faraday-cage }} \frac{d i}{d t}
$$

Any metal object around the explosives, such as a work stand, will also distort the electric fields. A computer simulation of the electric fields in a facility with a work stand and HE container is shown in Figure 1.3. In this configuration, the work stand is grounded, and the intensity of field around the $\mathrm{HE}$ (denoted in dark blue) is reduced relative to the rest of the work bay (denoted lighter blue). The area above work stand posts has much higher fields indicated by red. The fields on top of the container are also affected. Without an understanding of how the electric fields are

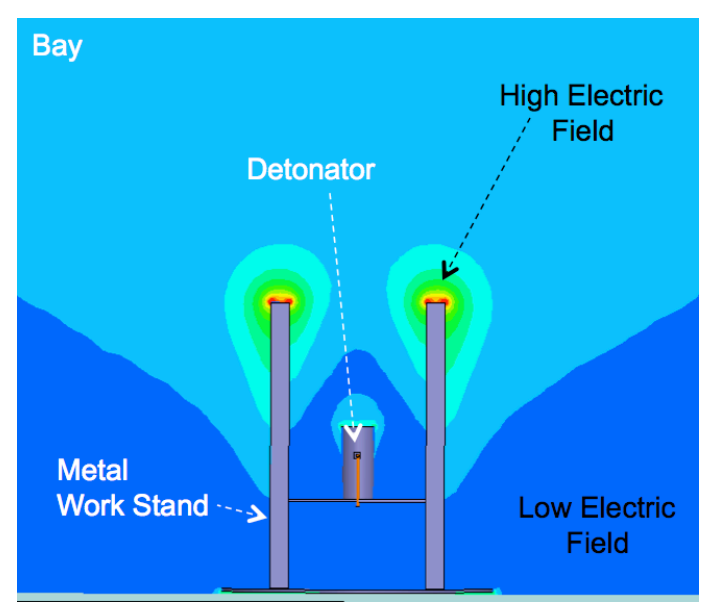

Figure 1.3. Metal work stands will distorted the electric fields. distributed near the detonator cable and container, it is not possible to calculate the induced detonator voltage.

The average lightning current has rise- and fall-times of 3 us and 50 us respectively, and this translates to a wavelength that is long when compared with the length of the HE container or 
detonator cable [1.2 - Brown]. Therefore, a simpler quasi-static analysis, where the propagation time is not tracked, is sufficient for computing voltages in the detonator.

As a simplification in this report, only the peak electric fields and peak voltages will be calculated rather than the complete temporal waveforms. The peak voltage is sufficient to establish if an arc will start to form. The calculation to determine the arc energy is much more involved and is not covered in this report [1.3 - Tully].

The goal of this study on unconventional dipoles is to validate the accuracy of existing standard antenna equations, and validation of the electromagnetic coupling codes. The equations are used to determine what configurations create the most stress. If the estimated voltages are of concern, computer simulations are performed to accurately establish induced voltages. Computer modeling is the main tool for the safety analysis, and therefore the validation is important. The validation process includes laboratory measurements of unconventional dipole antennas.

Other goals are to explain how electric fields are focused by the dipole, and the limitations of the standard antenna formulas. These equations will have inaccuracies if they are applied to unconventional dipoles beyond their intended range. The errors will be quantified, and are generally less than a factor of 2 when compared with computer modeling or laboratory measurements. The report is divided into three sections: (1) antenna equations, (2) computer simulations and (3) laboratory validation.

(1) The application of the antenna equations for monopoles and dipoles by an experienced RF analyst is a good starting point for estimating detonator voltages. If marginally high voltages are detected, then full electromagnetic (EM) computer simulations and circuit analyses should be completed to reduce uncertainty about the probability of a detonation.

Simulations are very time consuming because of the effort required to model all the surrounding metal structures and detonator components. This report covers only the first step of the safety analysis, estimating the detonator voltage using the antenna equations.

The plot in Figure 1.4 shows the antenna configurations that the three standard antenna equations for thin dipole, fat dipole, and monopole encompass. The canonical configuration in the illustration

Figure 1.4. Antenna equations were not intended for fat unbalanced dipoles like structures.
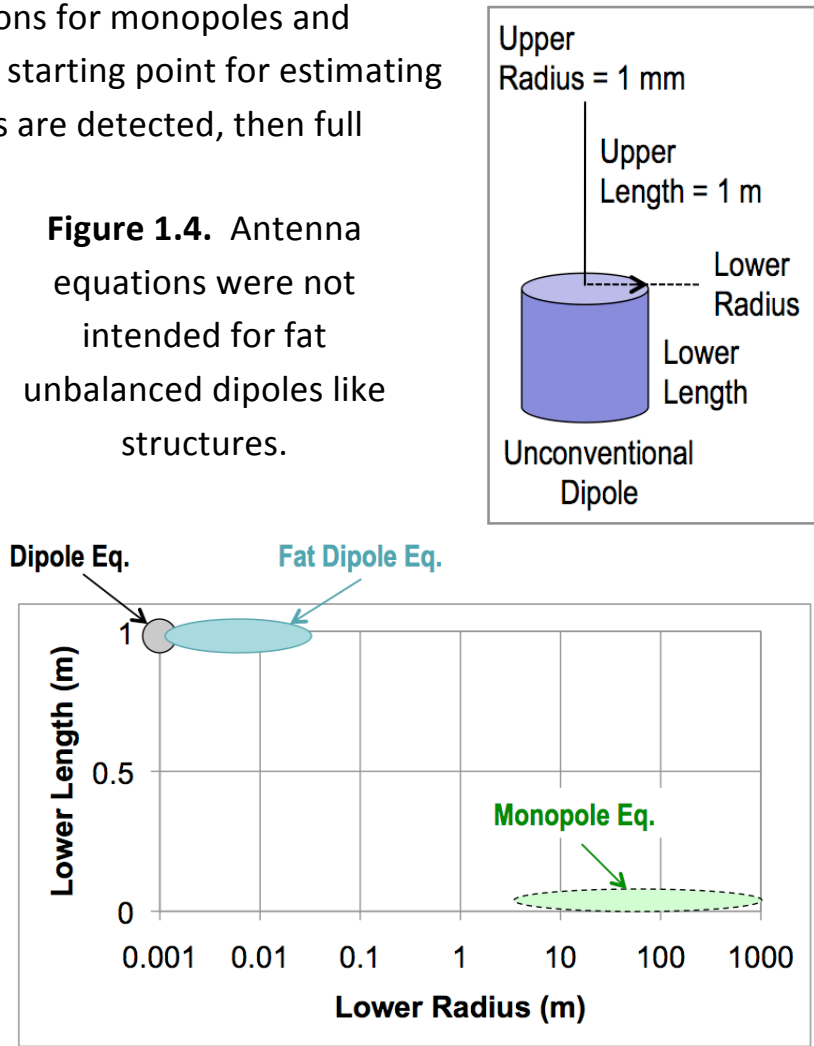
will be used later in the computer simulations of unconventional dipoles. The top arm is a typical thin element with a length of $1 \mathrm{~m}$ and radius of $1 \mathrm{~mm}$. In an HE safety analysis, the upper arm could correspond to the detonator cable. The lower arm could be shorter and wider which could represent an HE container and/or work stand support. The dipole equation assumes the bottom arm is $1 \mathrm{~m}$ long and thin. The fat-dipole equation assumes that the arm length-to-radius ratio is at least 17 [1.4 - Schmitt]. If the bottom radius is much wider than the upper element length, the lower arm will appear to be a "ground" plane, and the monopole equation applies. The monopole equation along with loading was validated in a previous study [1.5 - Crull]. A technical goal of the dipole study is to offer information that fills in the space in the above plot between the areas covered by the three equations.

While it is possible to analytically solve the unconventional dipole equation, the resulting complex formula would be difficult to interpret. In safety analysis, simple and somewhat less accurate equations are preferable to complex accurate equations that are difficult to check. The inaccuracies do not negatively impact safety because the simple formulas are on the conservative side. If there is any doubt about the application, a safety factor is added to compensate for uncertainties. Computer modeling will reduce uncertainty and improve accuracy.

(2) The computer simulations are broken into two parts: Electromagnetic coupling and circuit modeling of voltage loading. The dipoles will be excited by a nominal electric field and a graphic display of the field distortion will offer an intuitive understanding of the coupling. A parametric study will fill in the blank space in the plot in Figure 1.4. The RF dipole simulations will be based on 3D electro-static models that can easily run on a desktop computer. For simple dipole configurations, computer simulations produce results more quickly than laboratory experiments. Regardless, experimental results from interesting geometries will be compared with the computer simulations as a part of the validation process.

A circuit model, using the dipole voltages from the EM simulations as the input, will be used to account for the loading effect, such as from a laboratory instrument used to measure voltages. This circuit model also needs to be validated because it may be needed to model the electrical loading by the detonator cable. Typically loading will significantly reduce the voltage inside the detonator.

For efficiency reasons, loading effects are simulated with a circuit model rather than a 3D electromagnetic code. The dimensional difference between small objects like the bridge wire and the large $\mathrm{HE}$ container puts too much demand on the computer memory and processor. Instead, sub-structures are represented by lumped electrical elements in a circuit model. (In a safety analysis, a second analyst would check the EM and circuit simulations with analytical equations.) A detailed circuit model is useful for estimating the arc energy.

(3) An accurate laboratory validation can be difficult for four reasons. First, while an electrical field can be created in a Transverse Electromagnetic (TEM) Cell [1.6 - Crawford], the fields are not perfectly uniform. The spatial imperfections must be considered when exciting the 
dipole and analyzing the data. Second, the dipole voltage must be measured, and yet the measurement instrumentation, even just a cable with metallic wires, will distort the electric fields. Third, any electrical load, such as the instrumentation cable and digitizer, will reduce the dipole voltage. The loading effects in the experiment must be considered in the measurement design and included in the analysis of the data. Fourth, the laboratory study uses relatively minute electric fields when compared with lightning generated fields. Therefore, the laboratory dipole voltages will be small, and extra care is necessary to produce accurate measurements.

In a high-confidence safety analysis, validated electromagnetic simulation software is required to calculate lightning induced detonator voltages. In the validation process results from antenna equations, computer modeling and laboratory measurements are compared and should be consistent. If there are differences, they must be clearly explained. For a safety analysis, the facility and explosive device may not be available for RF coupling measurements or high-power testing to establish detonation voltage levels. In spite of that, application of the validated analytical and modeling tools is sufficient for an indirect-lightning safety analysis. (See Figure 1.5.)

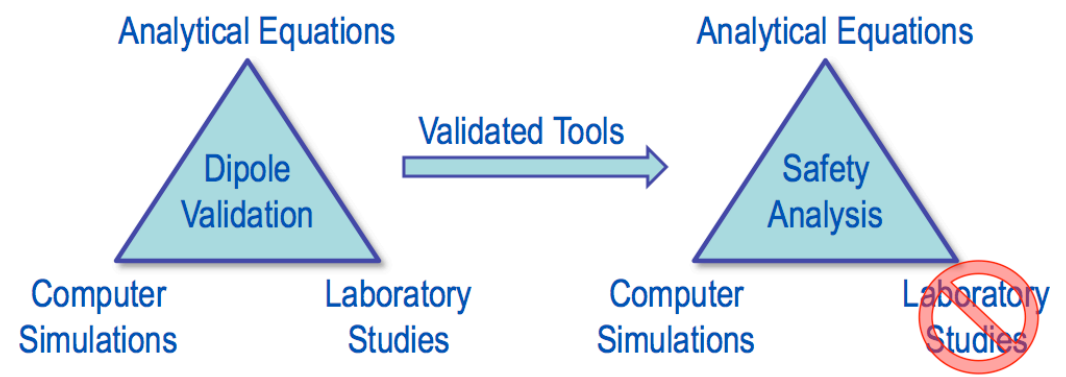

Figure 1.5. Validated tools based on dipoles can be applied in safety analyses of detonators when exposed to indirect lightning. 


\section{Antenna Equations}

This section is divided into three parts: (1) the basic monopole and dipole antennas equations, (2) the fat monopole equation and (3) antenna impedance and loading.

\subsection{Basic Antenna Equations}

The induced antenna voltage for a monopole or dipole is equal to the dot product of the electric field and the effective height, $h_{\text {eff, }}$ of the antenna. For short antennas, relative to the long lightning current wavelength, the effective height is about half the physical length of the antenna. The factor of two will become clear when the computer simulations are presented showing the concentration of the fields at the tips of the arms. In the example shown in Figure 2.1.1, a 1meter monopole in a uniform $1 \mathrm{~V} / \mathrm{m}$ electric field produces $0.5 \mathrm{~V}$ when the electric field is directly along the antenna [2.1 - Krause].

$$
\begin{aligned}
V_{\text {monopole }} & =E \mathrm{~h}_{\text {eff }} \approx E \frac{\mathrm{L}_{\text {monopole }}}{2} \\
\text { for } E=1 \mathrm{~V} / \mathrm{m} \text { and } L_{\text {monopole }}=1 \mathrm{~m} & \\
V_{\text {monopole }} & =1 \mathrm{~V} / \mathrm{m} * \frac{1 \mathrm{~m}}{2}=0.5 \mathrm{~V}
\end{aligned}
$$

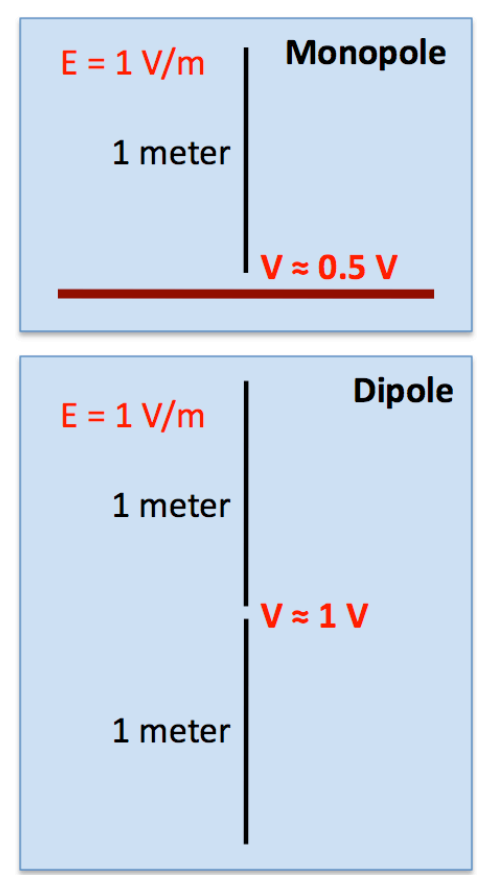

Figure 2.1.1. Antenna voltage depends on the field and arm length.

For the report, the assumption is that the electric field is vertically polarized and aligned with the antenna. It is also understood that the arms are very thin for the above formulas. The next part deals with so called "fat" antennas.

\subsection{Fat Dipole}

The balanced fat-dipole equation (2.3) is difficult to interpret because of its complexity, and in general, the effective height will decrease slightly with small increases in radius [2.2 - Schmitt]. Near the lower limit where the radius goes towards zero, the effective height matches the simple dipole equation, Eq 2.2, i.e., half of the total length. The fat-dipole equation can also be applied to a fat monopole antenna by removing the factor of two. 
The effective height of a 1-meter monopole as a function of width is shown in Figure 2.2.1. When the radius is infinitesimally thin the antenna has an effective height of 0.5 meter. The fat formula is thought to be valid for antenna configurations with a length-to-radius ratio of 17 or greater. The plot also shows $h_{\text {eff }}$ beyond the $59 \mathrm{~mm}$ upper radius limit, and those larger radii will be compared later in the simulation section.

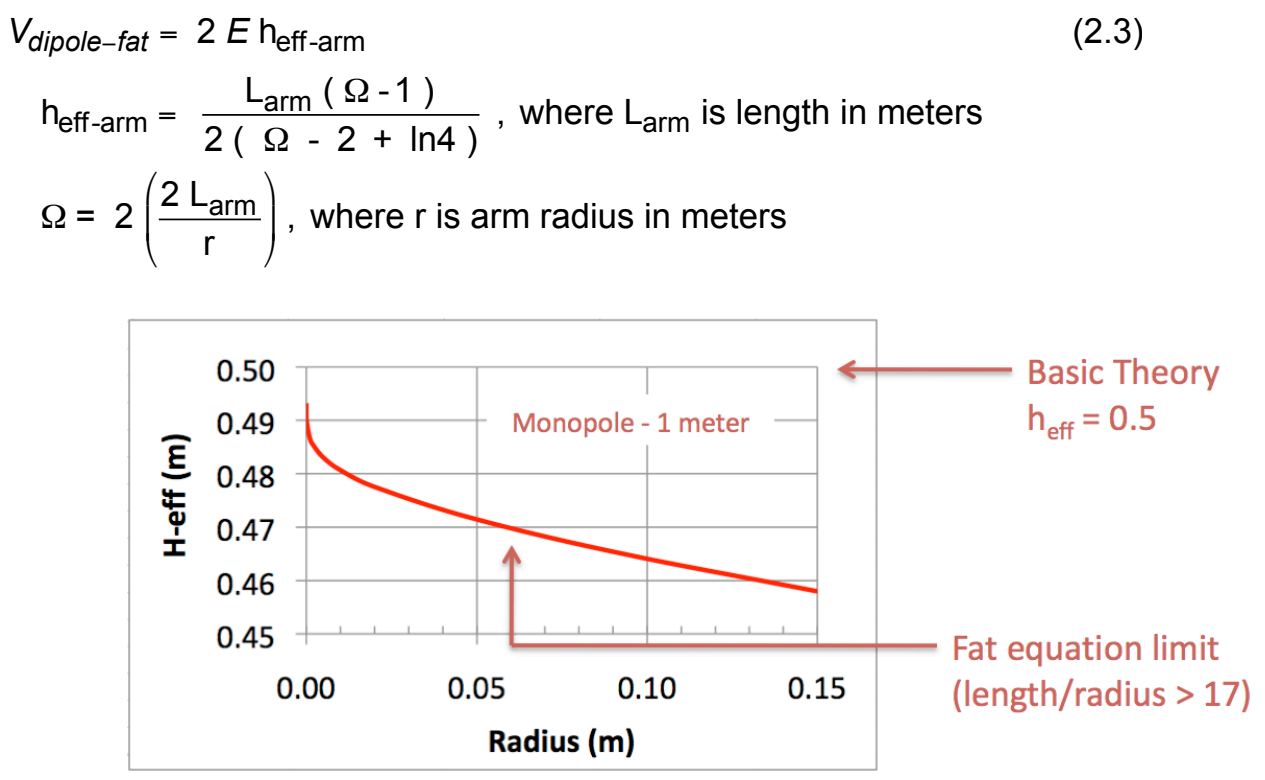

Figure 2.2.1. The effective height of a dipole antenna decreases slightly with increased radius.

For the lightning-coupling problem with our type of HE objects, the radius of one arm is much larger than appropriate for the fat equation. An alternate solution will be offered in the simulation section.

\subsection{Antenna Loading - Capacitive Divider}

So far only the voltage generated by a monopole or dipole antenna without loading, the open-circuit voltage, has been calculated. The antennas in the previous examples have modest output impedance at lightning current frequencies. Therefore even modest loading will reduce the detonator voltage. From a safety viewpoint, this could be an important phenomenon to consider in the analysis. Antenna loading increases the voltage safety margin.

We will explain the model starting with a loaded monopole [1.5 - Crull]. Later, the dipole model is created by combining the components from two monopoles. The portion of the detonator cable not exposed to RF and the detonator creates the load. For indirect-lightning RF coupling into detonators, only capacitive elements, the antenna $\left(\mathrm{C}_{\text {monopole }}\right)$ and load $\left(\mathrm{C}_{\text {load }}\right)$ are required in the model to calculate voltages. (See Figure 2.3.1.) The inductive impedance has little effect on the voltage at the low frequencies associated with lightning current and is not included in the model. For this report, it is assumed that voltage breakdown does not occur, and that the load resistance will be very high and is also neglected in the circuit model. The series 
resistance of the wire is also eliminated for the same reason; it is much lower than the antenna and load impedances.

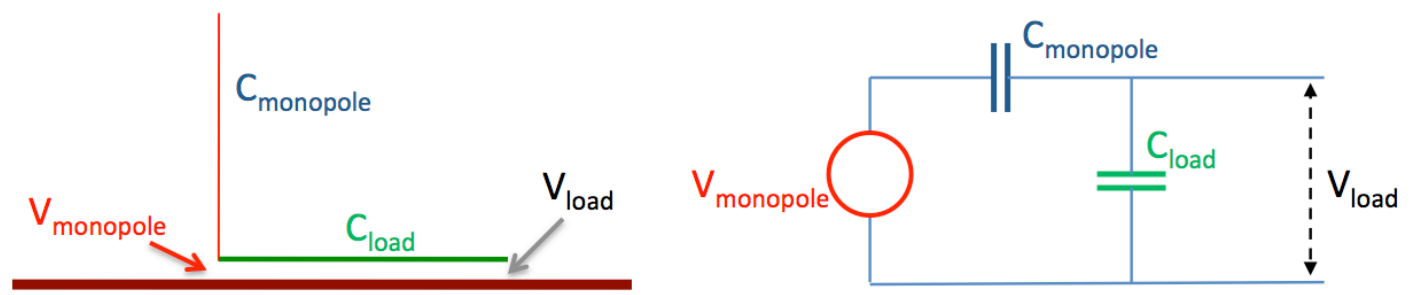

Figure 2.3.1. Capacitive circuit model for monopole antenna with loading.

The load voltage, $\mathrm{V}_{\text {load, }}$ is lower than the monopole voltage, $\mathrm{V}_{\text {monopole, }}$, because of the capacitive divider, Eq. 2.4. The monopole capacitance can be calculated from Eq. 2.5

$$
\begin{gathered}
V_{\text {load }} \approx V_{\text {monopole }} \frac{\mathrm{C}_{\text {monopole }}}{\mathrm{C}_{\text {monopole }}+\mathrm{C}_{\text {load }}} \\
V_{\text {monopole }}=E \mathrm{~h}_{\text {eff-monopole }}
\end{gathered}
$$

where $L_{\text {monopole }}$ is the length and $c$ is the speed of light [2.3 - Stutzman]. For a 1-meter monopole with a 1-mm radius, the capacitance is $9.4 \mathrm{pF}$. The impedance is 1.7 mega-ohms and 17 kiloohms at $10 \mathrm{kHz}$ and $1 \mathrm{MHz}$, respectively.

The load capacitance can be roughly estimated from standard parallel-plate capacitor equation 2.6 where $\varepsilon_{\mathrm{r}}$ is the relative dielectric constant, $\varepsilon_{0}$ is the permittivity, and $d$ is the separation distance between the two metal plates of equivalent area. The wire-over-a-ground-plane capacitance equation may be more

$$
\begin{aligned}
& \mathrm{C}_{\text {monopole }}=\frac{\mathrm{L}_{\text {monopole }}}{60 \mathrm{c}\left[\ln \left(\frac{\mathrm{L}_{\text {monopole }}}{\text { radius }}\right)-1\right]} \\
& \mathrm{C}_{\text {load-plate }} \approx \varepsilon_{r} \varepsilon_{O} \frac{\text { area }}{\mathrm{d}_{\text {separation }}} \\
& \mathrm{C}_{\text {wire-over-plane }} \approx \frac{2 \pi \varepsilon_{r} \varepsilon_{O}}{\ln \left(\frac{\text { height }}{\text { radius }}\right)}
\end{aligned}
$$

accurate but is more complex and less

intuitive [2.4 - Inan]. Calculating precise capacitance value involving complex structures requires 3D computer simulations that will include the fringe fields.

The dipole model shown in Figure 2.3.2 consists of components from two monopoles voltages and antenna capacitance as well as a load capacitance. The antenna components values can be estimated using equations 2.4 to 2.6. Assuming both arms see the same electrical fields, the voltage sources are in phase. 

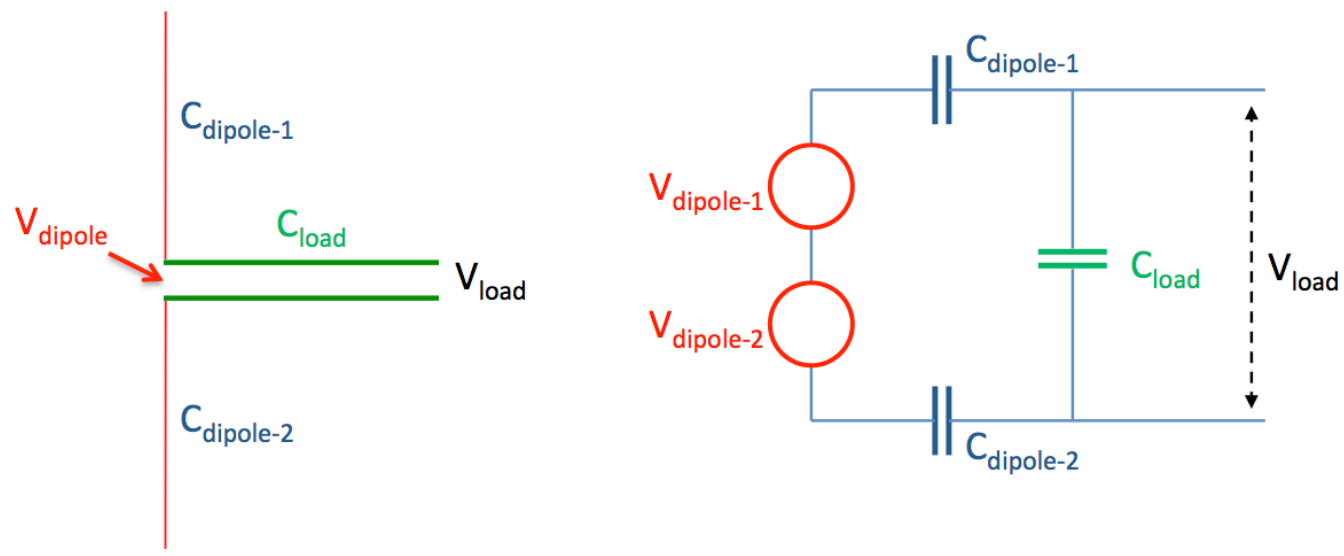

Figure 2.3.2. Capacitive model for dipole antenna using the monopole model with loading.

The double monopole model can be simplified to a simple dipole model shown in Figure 2.3.3. The loaded voltage can be computed from equation 2.7. Note that the two antenna capacitors when combined in series is less than for a 1-meter monopole. For a 2-meter dipole, the combined capacitance from the two arms is $4.7 \mathrm{pF}$. The dipole impedance is 3.4 mega-ohms and 34 kilo-ohms at $10 \mathrm{kHz}$ and $1 \mathrm{MHz}$, respectively, which is twice the impedance for the shorter 1-meter monopole. While the 2-meter dipole is an efficient voltage generator, it would deliver lower peak current than the smaller 1-meter monopole into a low impedance load. The calculated dipole and load voltages will be checked against computer modeling and laboratory study results.

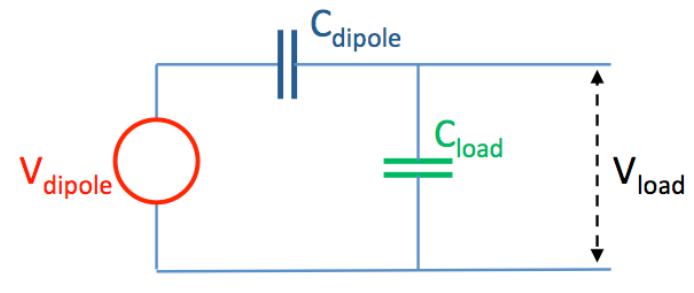

$$
\begin{gathered}
V_{\text {load }}=V_{\text {dipole }} \frac{\mathrm{C}_{\text {dipole }}}{\mathrm{C}_{\text {dipole }}+\mathrm{C}_{\text {load }}} \text {, where } \\
V_{\text {dipole }}=V_{\text {dipole-1 }}+V_{\text {dipole-2 }} \\
\mathrm{C}_{\text {dipole }}=\frac{\mathrm{C}_{\text {dipole-1 }} \mathrm{C}_{\text {dipole-2 }}}{\mathrm{C}_{\text {dipole-1 }}+\mathrm{C}_{\text {dipole-2 }}}
\end{gathered}
$$

Figure 2.3.3. The load voltage is reduced from the dipole voltage in the capacitive divider model.

A summary for the circuit characteristics of a 1-meter monopole and 2-meter dipole antenna

\begin{tabular}{|c|c|c|c|c|c|}
\hline Type & \begin{tabular}{l}
\multicolumn{2}{c}{ Size } \\
length radius
\end{tabular} & Voltage & Capacitance & $\begin{array}{c}\text { Impedance } \\
(10 \mathrm{kHz})\end{array}$ & $\begin{array}{c}\text { Impedance } \\
(1 \mathrm{MHz})\end{array}$ \\
\hline Monopole & $1 \mathrm{~mm}$ & $4.9 \mathrm{~V}$ & $9.4 \mathrm{pF}$ & $1.7 \mathrm{M} \Omega$ & $17 \mathrm{k} \Omega$ \\
\hline Dipole & $2 \mathrm{~m} \quad 1 \mathrm{~mm}$ & $9.7 \mathrm{~V}$ & $4.7 \mathrm{pF}$ & $3.4 \mathrm{M} \Omega$ & $34 \mathrm{k} \Omega$ \\
\hline
\end{tabular}
is given in Table. 2.3.1. The voltages were calculated with the fat antenna equation excited by an electric field of $1 \mathrm{~V} / \mathrm{m}$.

Table 2.3.1. Calculated antenna voltages and capacitances for circuit-model components. 


\section{Computer Modeling}

In this section, 3D electro-static simulations will be used to determine the antenna voltages generated by various dipoles. Electro-static simulations can be performed rather than full electromagnetic modeling because of the quasi-static approximation. The simulation software, MAXWELL v12, is from Ansoft Corporation [3.1], and it ran on a personal computer with Microsoft Windows XP. The three dimensional simulation is not needed for the dipole validation because of the symmetry; 2D is sufficient. However, the 3D version of the EM code is needed in the safety analyses of complex structures, and therefore needs to be a part of the validation.

The physical parameters of the dipole are shown in Figure 3.1. The baseline dipole is 2meters tall with 1-mm radius arms, and a 1-cm gap between the arms. Four parameters will be studied: lower arm radius (fat), lower arm length (unbalanced), gap separation between the arms (a component of capacitive loading), and distance above the ground plane. In the electromagnetic simulations, no loads will be attached to the dipoles. Loading effects are usually evaluated with circuit models and are discussed at the end of the section.

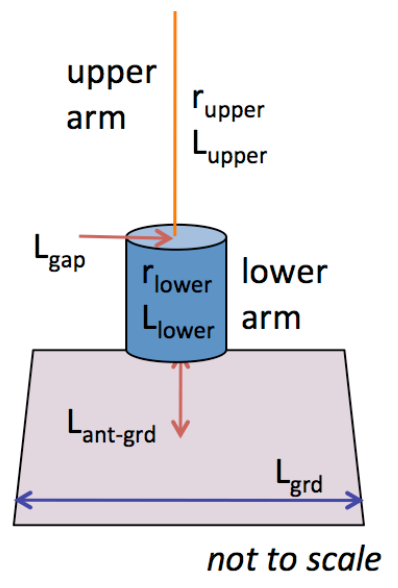

\begin{tabular}{|l|c|c|l|}
\hline Parameter & Variable & Range & Note \\
\hline Ground plane & $\mathrm{L}_{\text {grd }}$ & $12 \times 12 \mathrm{~m}$ & fixed \\
\hline Radius - upper & $\mathrm{r}_{\text {upper }}$ & $1 \mathrm{~mm}$ & fixed \\
\hline Length - upper & $\mathrm{L}_{\text {upper }}$ & $1 \mathrm{~m}$ & fixed \\
\hline Radius - lower & $\mathrm{r}_{\text {lower }}$ & $1 \mathrm{~mm} \rightarrow 5 \mathrm{~m}$ & Fat \\
\hline Length - upper & $\mathrm{L}_{\text {lower }}$ & $1 \mathrm{~m} \rightarrow 1 \mathrm{~m}$ & Unbalanced \\
\hline Gap antenna base & $\mathrm{L}_{\text {gap }}$ & $0.1 \mathrm{~mm} \rightarrow 100 \mathrm{~mm}$ & \\
\hline Distance ant to grd & $\mathrm{L}_{\text {ant-grd }}$ & $0 \mathrm{~mm} \rightarrow 2 \mathrm{~m}$ & \\
\hline
\end{tabular}

Figure 3.1. Four parameters will be studied in the laboratory validation.

The simulation volume was chosen to be sufficiently large so that the $1 \mathrm{~V} / \mathrm{m}$ electric field is uniform at the edge of the volume. It is set up to be at least 12-meters deep by 12-meters wide, and 6-meters tall. (See Figure 3.2.) In the simulation space the dipole is too small to be seen in the display, but the concentrated electric fields around the tips of the dipole arms are visible as two red bulbs.

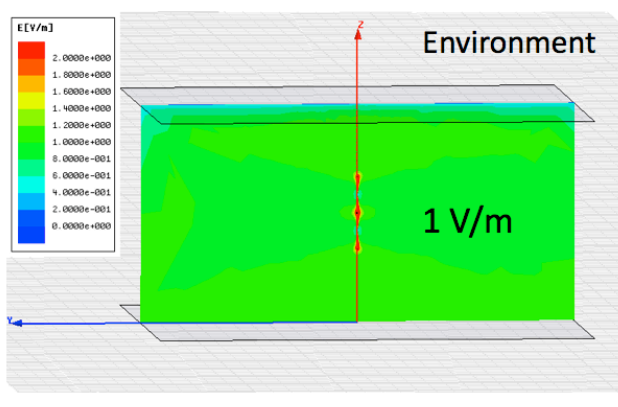

Figure 3.2. Simulation volume is large relative to dipole size.

Because of the coupling difference is sometimes subtle, high-resolution spatial models with low energy-error are required to calculate antenna voltages with less than $1 \%$ error. (See Figure 3.3.) MAXWELL uses an adaptive meshing technique to increase the number of 
tetrahedrons in high gradient areas, e.g., around the dipole. In addition, a vacuum box was added to the gap between the arms to increase the number of tetrahedrons so the antenna voltage could be more accurately computed. The simulation starts by creating a mesh with a small number of tetrahedrons, and the number is increased by the code over multiple iterations until the energy-error specification is met. A simulation typically completes in under an hour. The simulation requirements and typical mesh count are listed in Figure 3.3.

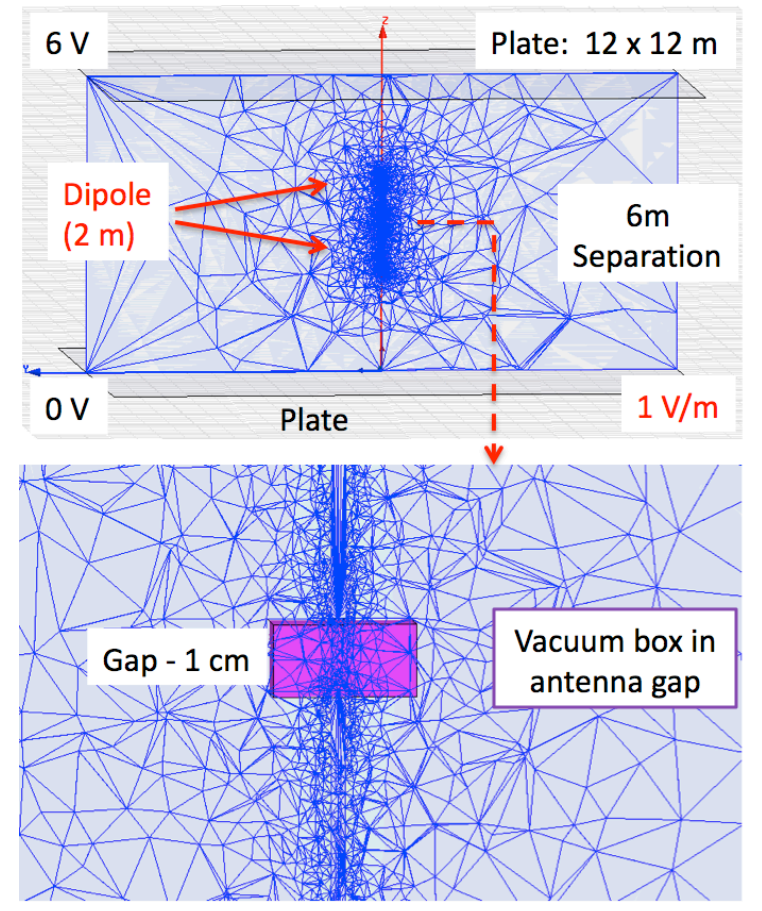

\begin{tabular}{|c|c|}
\hline Antenna Size & Value \\
\hline Radius & $1 \mathrm{~mm}$ \\
\hline Length-upper & $1 \mathrm{~m}$ \\
\hline Length-lower & $1 \mathrm{~m}$ \\
\hline Ant. Gap & $1 \mathrm{~cm}$ \\
\hline Model & Tetrahedrons \\
\hline Antenna & $2 \times 14 k$ \\
\hline Gap (box) & $3 k$ \\
\hline Volume & $200 k-600 k$ \\
\hline Simulation & Settings \\
\hline Passes & $\geq 23$ \\
\hline Energy Error & $10^{-4}$ \\
\hline Run Time & $<1$ hour \\
\hline
\end{tabular}

Figure 3.3. The mesh plot shows the fine details of the dipole model.

The electric field intensity for the baseline dipole is shown in the left plot in Figure 3.4. The blue background indicates $1 \mathrm{~V} / \mathrm{m}$. The red color designates a higher field region. The dipole voltage is calculated along a vertical line in the gap aligned with the long axis of the antenna using the Maxwell voltage calculator, Eq. 3.1. (See right plot.) As expected the dipole voltage is slightly less than $1 \mathrm{~V}, 0.966 \mathrm{~V}$. The user is allowed to set $\mathrm{n}$, the number of steps, in the calculator. A large number is needed if the field gradient changes quickly. Typically a hundred steps are selected.

$$
V_{\text {gap }}=\frac{1}{2} \sum_{\mathrm{k}=1}^{\mathrm{n}} E_{z, k} \Delta \mathrm{z}_{\mathrm{k}}, \mathrm{n} \geq 100
$$




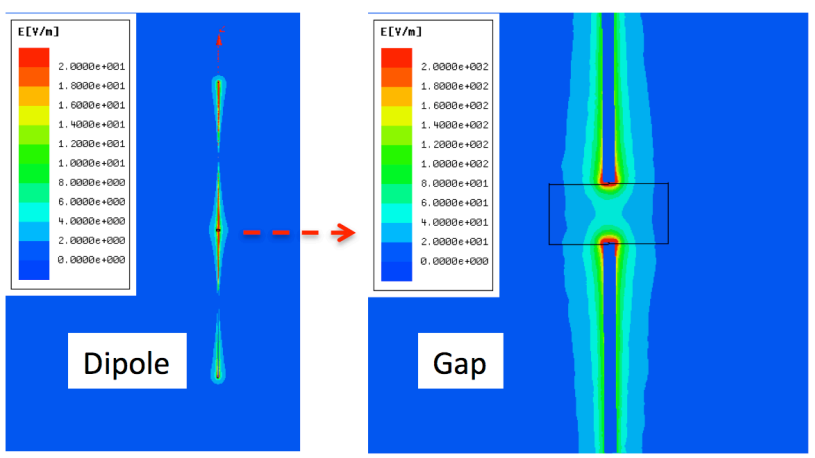

- Base line:

- ant length $=2 \times 1 \mathrm{~m}$

- ant radius $=1 \mathrm{~mm}$

- ant gap $=1 \mathrm{~cm}$

- Antenna gap voltage:

- line along z-axis

- use Maxwell field calculator

- gap voltage: $0.966 \mathrm{~V}$

Figure 3.4. The electric fields around the baseline dipole produce almost $1 \mathrm{~V}$ in the gap.

\subsection{Fat Dipole}

A typical fat-dipole configuration with an expanded lower arm is shown in the left plot of Figure 3.1.1. The upper arm has the baseline configuration of 1-meter tall and with 1-mm radius. The lower arm length will also be kept at 1-meter. The electric field around the 1-cm gap between the arms is shown in the image on the right. The gap voltage will be calculated for various lower arm radii.
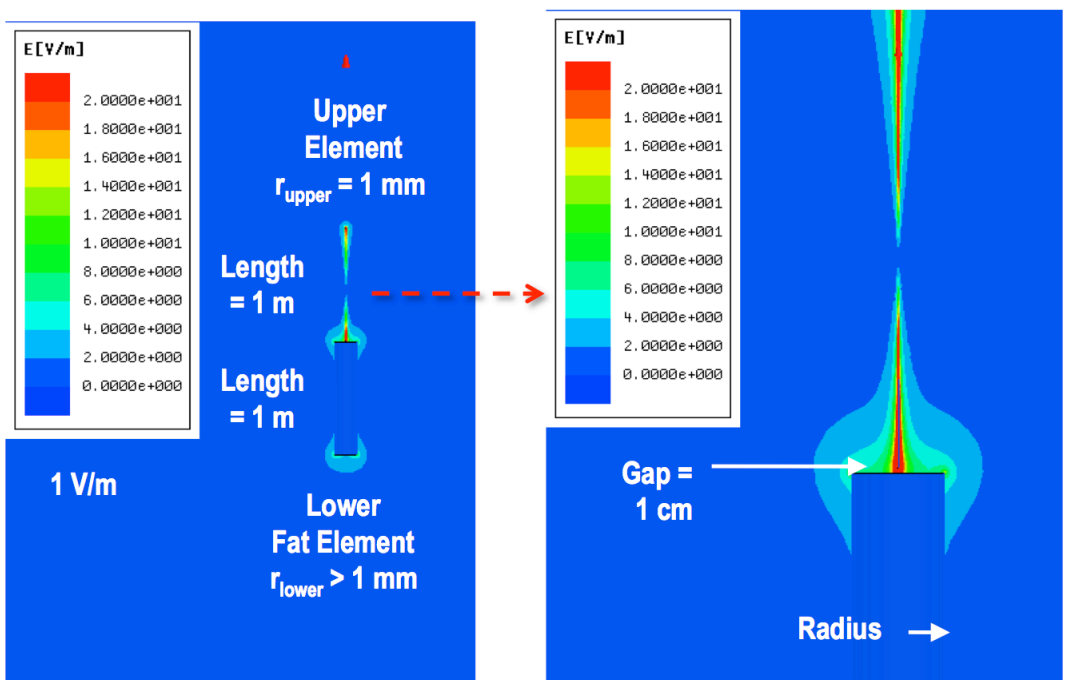

Figure 3.1.1. The electric field is very intense in the gap between the dipole arms.

In Figure 3.1.2, the results of the simulations are plotted (blue line) as well as the voltages from the antenna equations (red line). The simple dipole and monopole equations return $1.0 \mathrm{~V}$ and $0.5 \mathrm{~V}$ for the 2-meter and 1-meter configurations, respectively. The fat-dipole antenna voltage was computed by applying the fat monopole equation to each arm and adding together the two fat monopole voltages. In this example, except at smallest radius of the lower arm, the voltages from the fat-dipole equation were higher than results from the simulations.

$$
V_{\text {dipole }}=V_{\text {fat-upper }}+V_{\text {fat-lower }}
$$




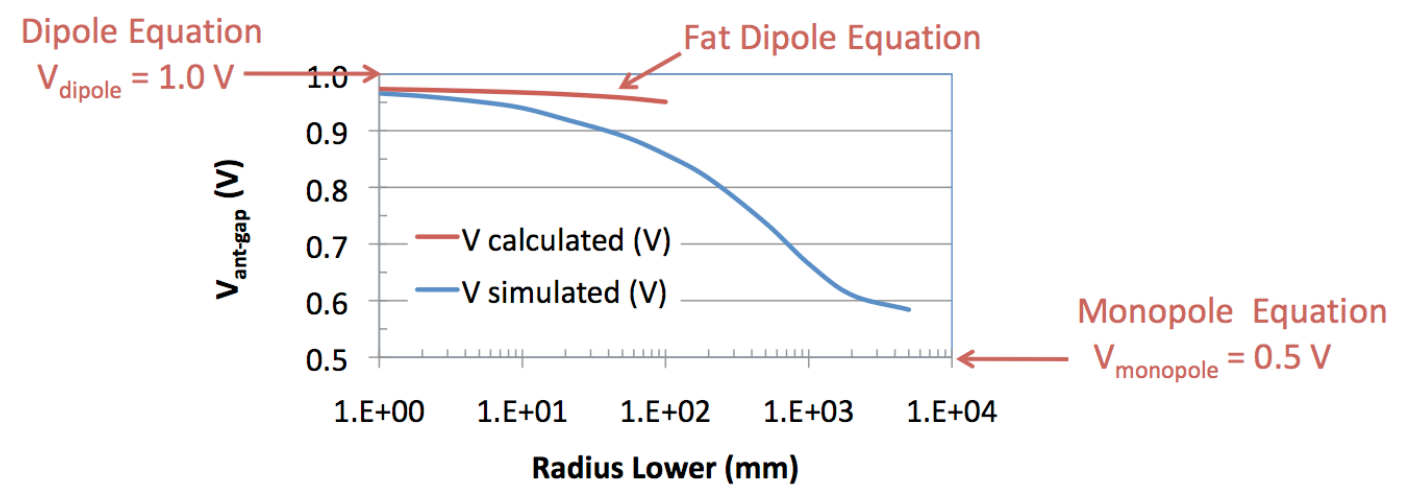

Figure 3.1.2. Comparison of modeling and antenna equation voltages for fat dipoles.

Beyond some point in the radius expansion, the lower fat dipole will appear as a traditional ground plane relative to the upper arm. In this monopole arrangement the gap voltage will be 0.5 $\mathrm{V}$. When the radius of the lower arm equals the height of the upper arm, the simulated voltage has drops to $0.67 \mathrm{~V}$. The phenomenon can be explained with the help of Figure 3.1.3. The nominal $1 \mathrm{~V} / \mathrm{m}$

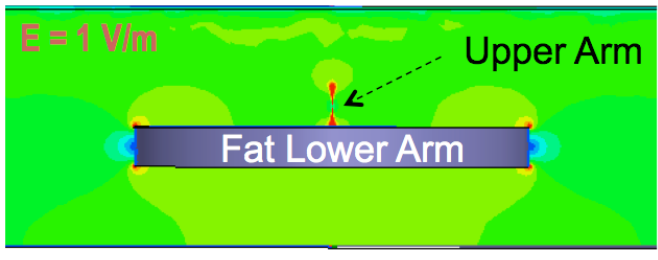

Figure 3.1.3. Very wide lower arm appears to be a ground plane to upper electric field is denoted in green. The upper arm draws the electric field into the base of the antenna. The concentrated fields associated with the lower fat arm are at the corners, well away from the base of the upper arm. Hence the lower arm appears to be a ground plane.

In a safety analysis where the fat dipole is floating, the dipole and monopole voltages bound the antenna voltage. There were no surprises with the fat-dipole voltage. If the lower arm is not floating, the voltage could be higher, and will be demonstrated later in the section.

\subsection{Unbalanced Dipole}

Representative unbalanced dipole arrangements are shown in Figure 3.2.1. The baseline radius of $1 \mathrm{~mm}$ and gap separation of $1 \mathrm{~cm}$ is unchanged. Note that the field around the short arm relative to the longer upper arm is smaller, indicating a smaller antenna capacitance. The lower fields also indicate that the lower element will contribute less to the gap voltage. The following analytical equation, Eq. 3.3, was used to calculate the unbalanced dipole voltage. The effective height was set to half of the total length. The simulated and calculated voltages started to diverge as the lower arm became shorter. (See Figure 3.2.2.)

$$
V_{\text {dipole-unbalanced }} \approx E \frac{\mathrm{L}_{\text {upper }}+\mathrm{L}_{\text {lower }}}{2}
$$



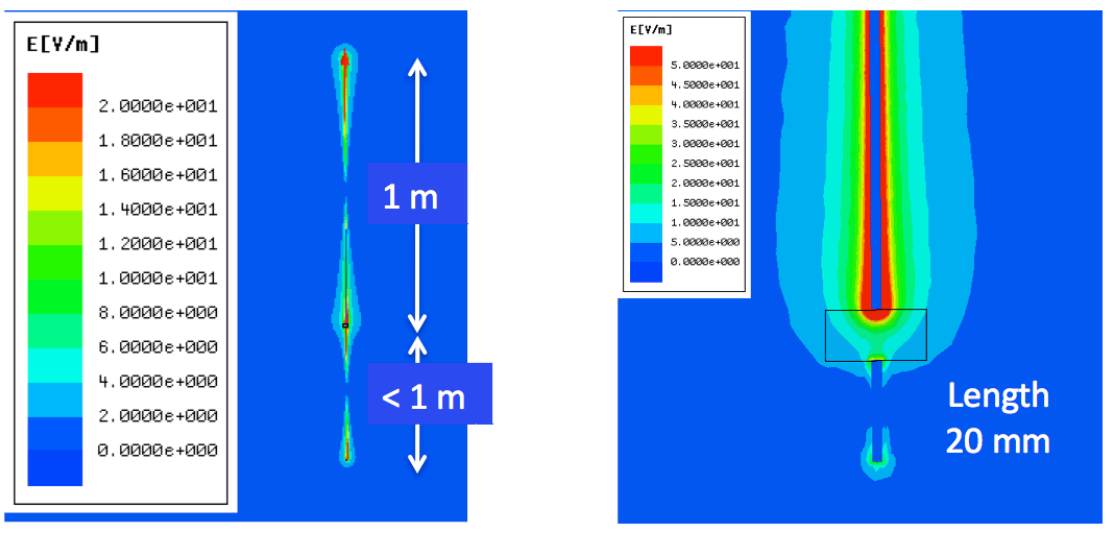

Figure 3.2.1. The shorter arm of the unbalanced dipole is exposed to a lower net surrounding field.

On the right edge of the plot, as expected, the antenna voltage is $1 \mathrm{~V}$ for the 2 -meter dipole. As the lower arm was shortened, the simulation produced lower voltages than predicted by the simple formula, dropping below $0.5 \mathrm{~V}$. At $20 \mathrm{~mm}$, the gap voltage from simulation using the MAXWELL calculator was $0.40 \mathrm{~V}$. The modeling result was checked a number of ways that included looking at the equal potential lines, and 2D model. (See Appendix A - Very High Resolution Models of Unbalanced Dipoles.)

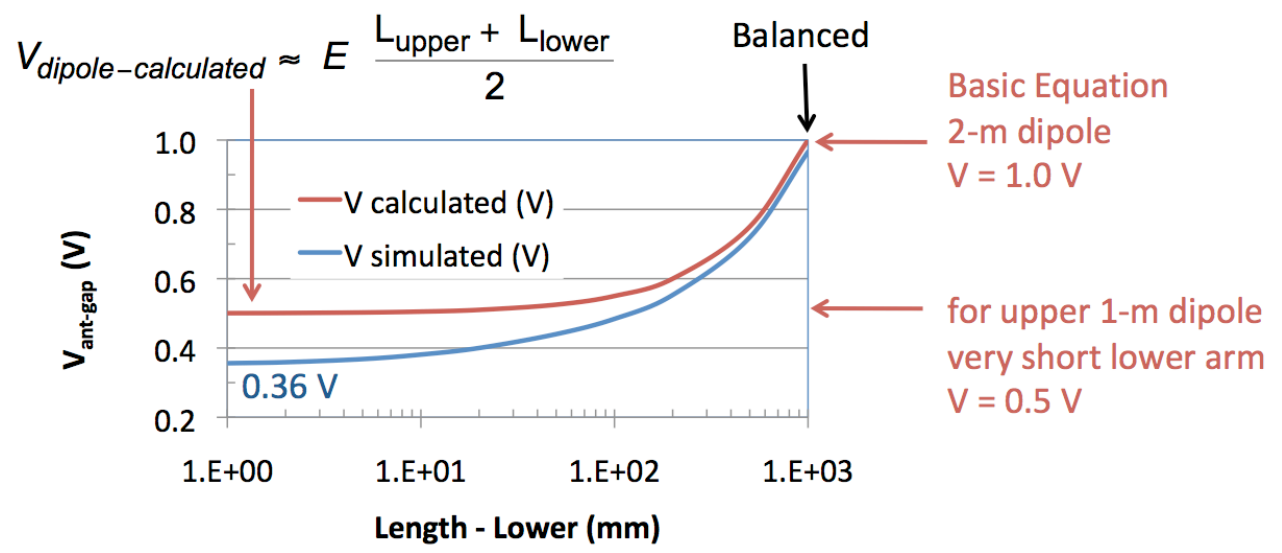

Figure 3.2.2. Computer simulations of an unbalanced dipole indicate a lower antenna voltage than from the standard formula.

The equipotential lines around the antenna gap are shown in Figure 3.2.3. The top portion of the mesh for the $20 \mathrm{~mm}$ lower arm is superimposed on the field map to illustrate the fine resolution. The upper arm is at a potential of $3.525 \mathrm{~V}$ relative to the ground plane for the simulation environment. The lower one is at $3.125 \mathrm{~V}$. The difference is $0.40 \mathrm{~V}$, which is the same potential produced by the MAXWELL calculator operating on the simulated fields plotted in Figure 3.2.2. Even when capacitive loading of the gap was considered, the capacitive divider formula, Eq. 2.7, still predicted $0.40 \mathrm{~V}$. 

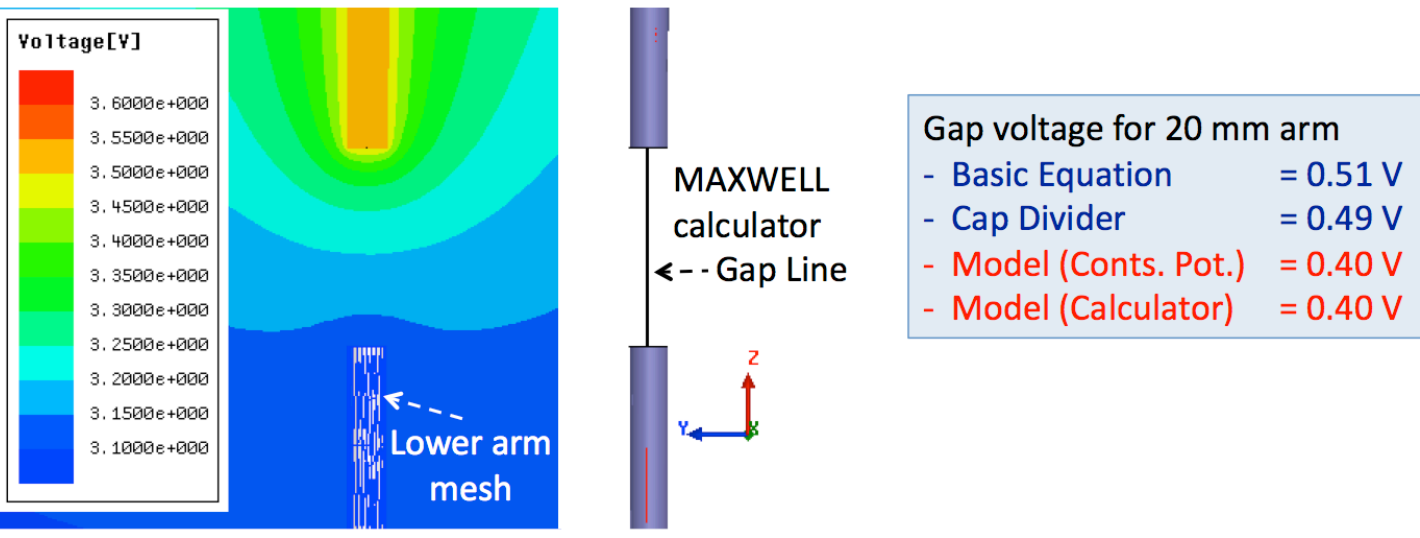

Figure 3.2.3. Equipotential lines indicate that the voltage between the arms is $0.40 \mathrm{~V}$.

The difference between the electro-static simulation and the formula is due to the electric field interaction, or mutual coupling, between the two arms. The upper arm distorts the field around the lower arm. The electric field associated with the upper arm is pushed down around the 20-mm element. This is visible in the right plot in Figure 3.2.1. Hence, a portion of the field that produced the $3.525 \mathrm{~V}$ on the upper arm also appears on the lower arm, raising the lower arm potential, and reducing the difference. The formula does not include this field distortion caused by the upper arm, or the mutual coupling. The lower arm also distorts the field around the bottom portion of the upper arm, but its shorter length leads to a minimal effect on the upper arm voltage.

The simulation was repeated in Appendix A starting with a higher resolution 2D model. The results were the same. 


\subsection{Gap Spacing and Loading}

In the previous two examples, the gap between the arms was set to $1 \mathrm{~cm}$ so that it would contribute very little to voltage loading. Two phenomena will be studied by varying the gap separation of the baseline dipole: capacitive loading caused by the gap, and the effect on the potential differences for widely separated arms. In Figure 3.3.1 the baseline $1 \mathrm{~cm}$ gap is shown. It will be reduced to illustrate capacitive loading and increased to quantify the effect of

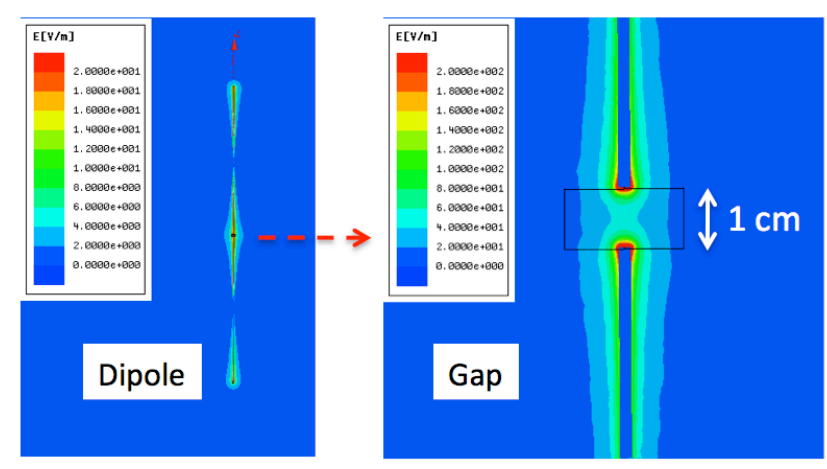

Figure 3.3.1. Gap spacing will be varied from 0.1 $\mathrm{mm}$ to $100 \mathrm{~mm}$ in the simulation. large gaps on voltage. While significant gaps may not exist in a detonator, this configuration could be representative of the effect of the work stand and cable separation on the detonator voltage.

The gap capacitance is estimated by Eq. 3.4. The actual capacitance may be slightly bigger

$$
\begin{array}{r}
C_{\text {gap }}=\varepsilon_{r} \varepsilon_{0} \frac{\mathrm{A}}{\text { gap }}, \varepsilon_{r}=1, \\
A=\pi \mathrm{r}^{2}, r=1 \mathrm{~mm}
\end{array}
$$
because of fringe fields not included in the equation. The dipole antenna capacitance equation, Eq. 3.5, with the total length, $2 \mathrm{~L}_{\text {arm, }}$, explicitly identified, is a variation of Eq. 2.5 for monopoles.

The calculated from Eq. 2.7 and simulated dipole voltages for various gap lengths are shown in Figure 3.3.2. The calculated dipole capacitance is about $4.7 \mathrm{pF}$. At the baseline gap of $1 \mathrm{~cm}$, according to the formula, the capacitive loading is insignificant. At a span of $1 \mathrm{~mm}$, the gap load capacitance is still very small, about $1 \%$ of the antenna capacitance. Hence there is little loading, causing a $1 \%$ drop in the calculated dipole voltage. As the gap closes further, the antenna voltage drops.

The simulated dipole voltage is lower than the calculated voltage when the gap is less than $30 \mathrm{~mm}$. The phenomenon of mutual coupling described in the previous short arm (unbalanced) example accounts

$$
\begin{aligned}
C_{\text {dipole }}= & \frac{2 L_{\text {arm }}}{2 c 120\left[\ln \left(\frac{2 L_{\text {arm }}}{2 r}\right)-1\right]}, \\
& L_{\text {arm }}=1 \mathrm{~m} \\
& c=310^{8} \mathrm{~m} / \mathrm{s}
\end{aligned}
$$

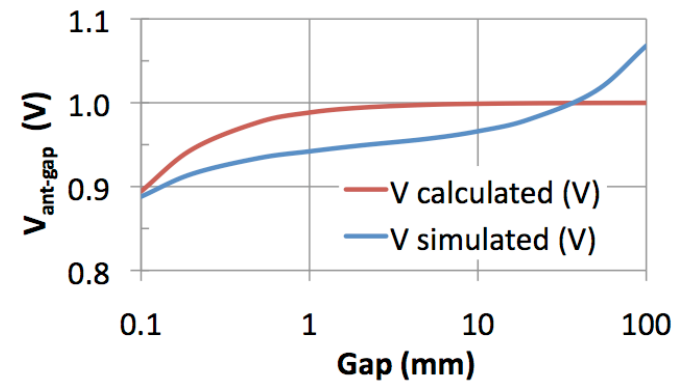

Figure 3.3.2. There are small differences between the calculated and simulated gap voltage. for some of the difference. The mutual coupling between the arms will depress the antenna voltage. This is a small effect, and the capacitive loading only dominates at the smallest gap separations, less than $1 \mathrm{~mm}$. 
The simulated dipole voltage is higher than the calculated voltage when the gap is more than $30 \mathrm{~mm}$. For a large gap, the formula to determine the antenna gap voltage must be amended to include the voltage contribution from the electric field in the gap.

$$
V_{\text {dipole-large-gap }}=V_{\text {dipole }}+\int_{\text {gap }} \vec{E} \cdot \mathrm{dl}
$$

As an illustration, consider the configuration with an exaggerated gap of 1 meter. The addition of the voltage from the gap field will produce results close to the simulation voltage. In Figure 3.3.3, the equipotential lines from the simulation allow the voltage difference between the two arms to be easily resolved. The upper arm is at $4.5 \mathrm{~V}$ relative to the ground, and the lower arm is at $2.5 \mathrm{~V}$. The difference is $2.0 \mathrm{~V}$, and is close to the simulation calculator voltage of $1.9 \mathrm{~V}$.

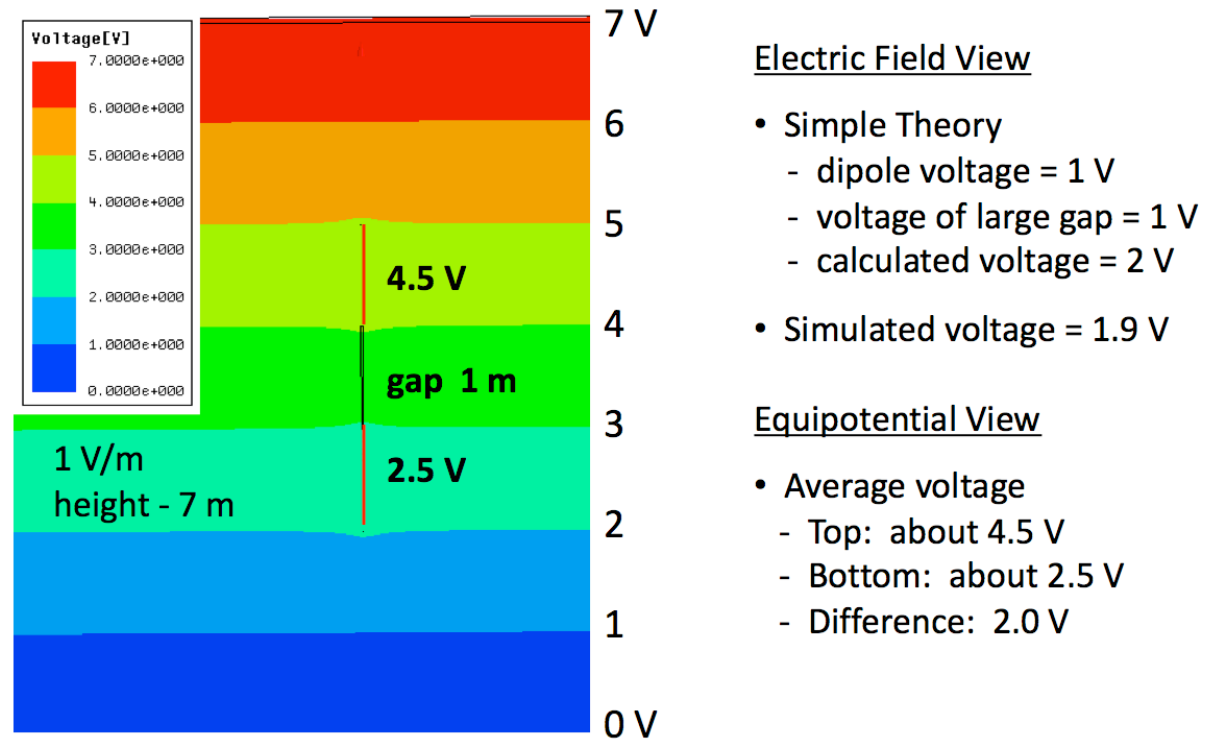

Figure 3.3.3. Equipotential-line view of voltage around dipole with a large 1-meter gap.

Any practical small gap size in a dipole has little effect on the open-circuit voltage of the antenna. The second term on the right side of Eq. 3.6 can be disregarded.

\subsection{Antenna-to-Ground Separation}

Sometimes an explosive device is assembled on a metal work stand, and the question arises weather or not the stand should be grounded. In the following simulation, from a RF coupling and safety perspective, the theoretical answer is no. Depending on the construction, electrically floating the stand could reduce the voltage in the detonator. There may be other reasons for grounding the stand, such as for electro-static protection. These arguments will not be covered in this report.

In Figure 3.4.1, the baseline dipole is positioned close to the ground plane. The voltage in the gap of the dipole antenna increases slightly as the antenna moves towards the ground. 
However, when the lower arm of the dipole makes electrical contact with the ground plane, the dipole voltage rises by about $50 \%$. (See Figure 3.4.2.)
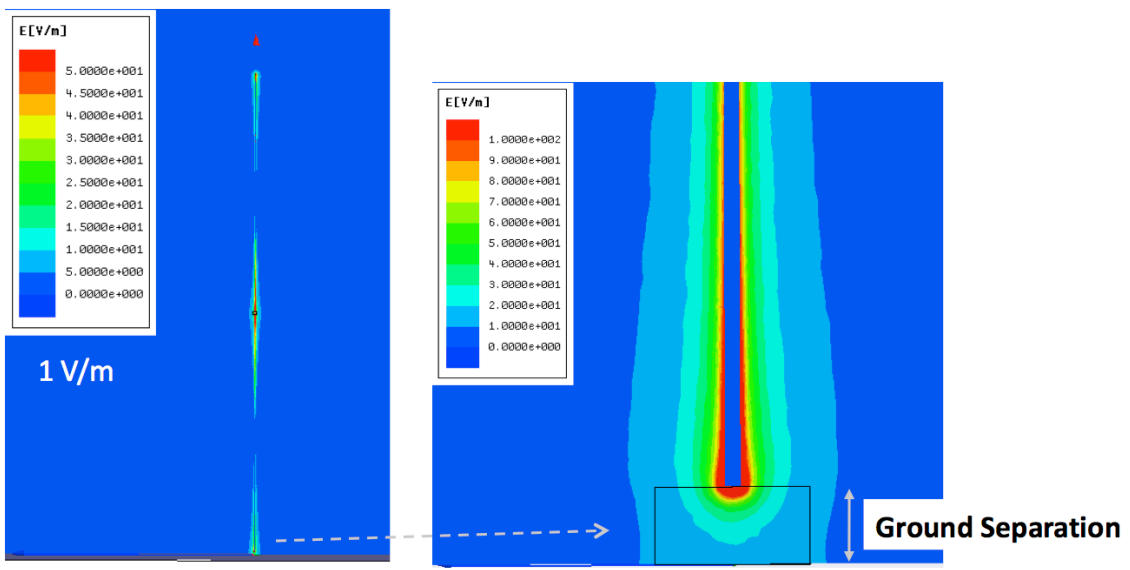

Figure 3.4.1. The dipole antenna is moved down towards the ground plane.

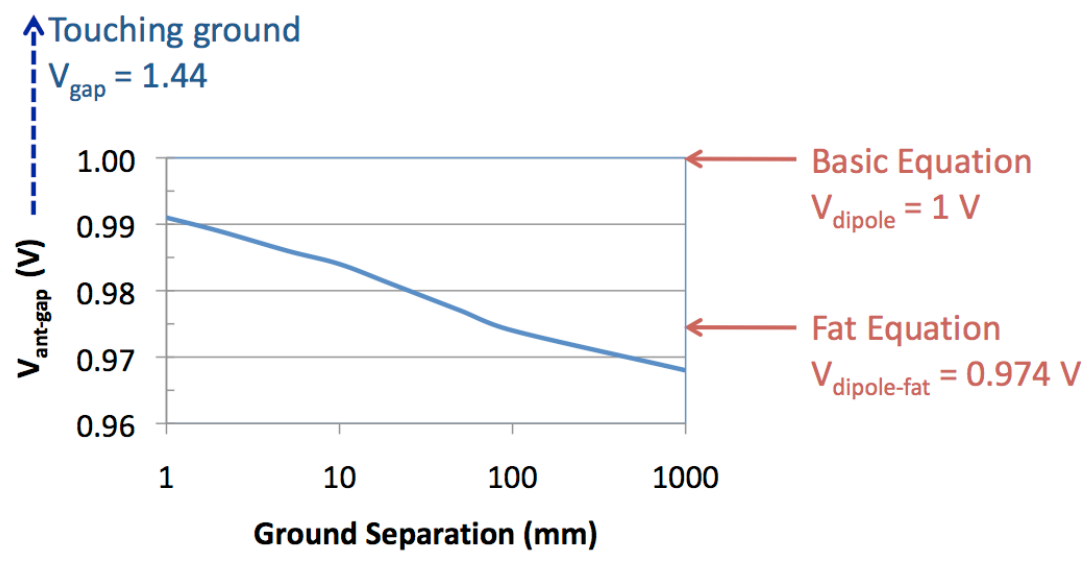

Figure 3.4.2. The dipole voltage increases slightly as the antenna approaches the ground plane until there is contact, then the voltage increases by about $50 \%$

The increase in voltage can be explained by comparing the electric field pattern around the dipole when there is separation from the ground plane and when touching. (See Figure 3.4.3) For an arm, the electric field is concentrated more or less equally at the upper and lower tips. On the vertical axis for the $1 \mathrm{~V} / \mathrm{m}$ environment, the voltage generated by the condensed fields at each tip is about $0.5 \mathrm{~V}$, or $25 \%$ of the total voltage, $2 \mathrm{~V}$. (This effect explains the factor of 2 in Eq. 2.2.) However, if the lower arm touches the ground plane, the electric field must go to zero on the lowest tip. In this grounded-monopole configuration, the potential difference that would have been between the lower arm and the ground is forced to the gap between the elements. In the example the gap voltage jumps from $1 \mathrm{~V}$ to $1.5 \mathrm{~V}$. 


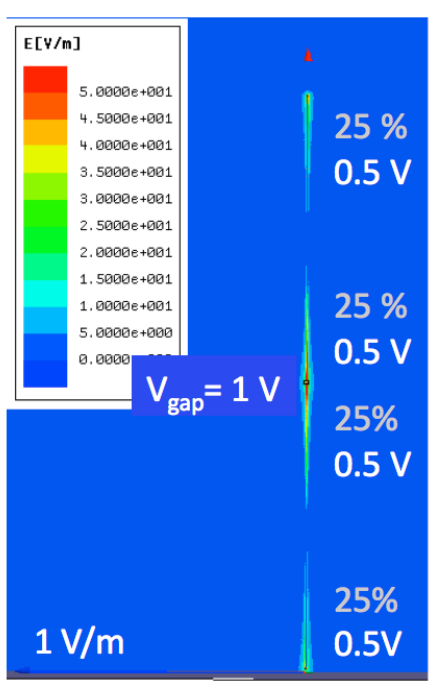

Ground Separation

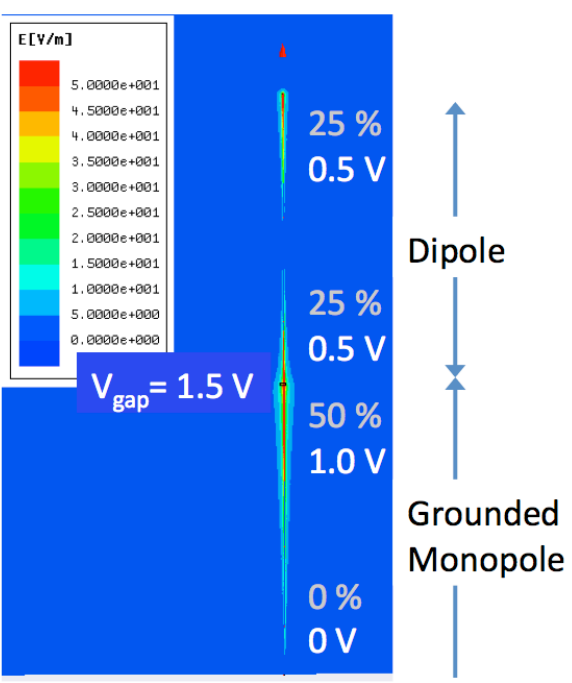

Touching ground

Figure 3.4.3. Field distribution for dipole almost touching and touching ground plane.

\subsection{Circuit Modeling of Antenna Loading}

The previous examples have focused on the antenna and not on the electrical load that normally consists of the unexposed detonator cable and the detonator. The most efficient tool for computing the loaded detonator voltage is a circuit simulator. The model includes all the RF elements: sources, capacitors, inductors and resistors. In practice the capacitance components establish the loading of the antenna voltage.

The capacitances can be calculated from equations or more precisely from static EM computer models. For the antenna capacitance, the dipole elements are excited by a voltage source $\left(V_{\text {dipole }}\right)$, and the simulator calculates the energy stored in the electric field (Energy electric- $_{\text {- }}$ field) around the antenna. The following equation is used to calculate the dipole capacitance $\left(C_{\text {dipole }}\right)$ :

$$
\text { Energy }_{\text {electric-field }}=\frac{1}{2} \mathrm{C}_{\text {dipole }} \mathrm{V}_{\text {dipole }}^{2}
$$

Computer modeling is especially important for unconventional dipoles where the equations may be less accurate. The load capacitances can also be determined by the EM computer models.

In the circuit model in Figure 3.5.1, the values of the inductive and resistive components are difficult to read, are not important for understanding dipole antennas at low frequencies, and will be different for each application, depending on detonator cable types and position, and HE container geometries. There are two antenna paths to signify the two leads in the detonator cable. The temporal shape of the voltage source waveform follows the derivative of lightning current, and the amplitude was calculated from the electric field near the cable when a facility is struck by lightning. The source is driven from a table of voltages, and a filter was added to the source to reduce the quantization noise. The antenna capacitances can be calculated using 
either Eq.'s 2.5 or 3.5. The other capacitances, between the wires, for the exposed and shielded portion of the cable can be determined from an electro-static computer model. The potential difference of interest is between the cable wire and some grounded metal object, e.g., a drumshaped object and work stand.

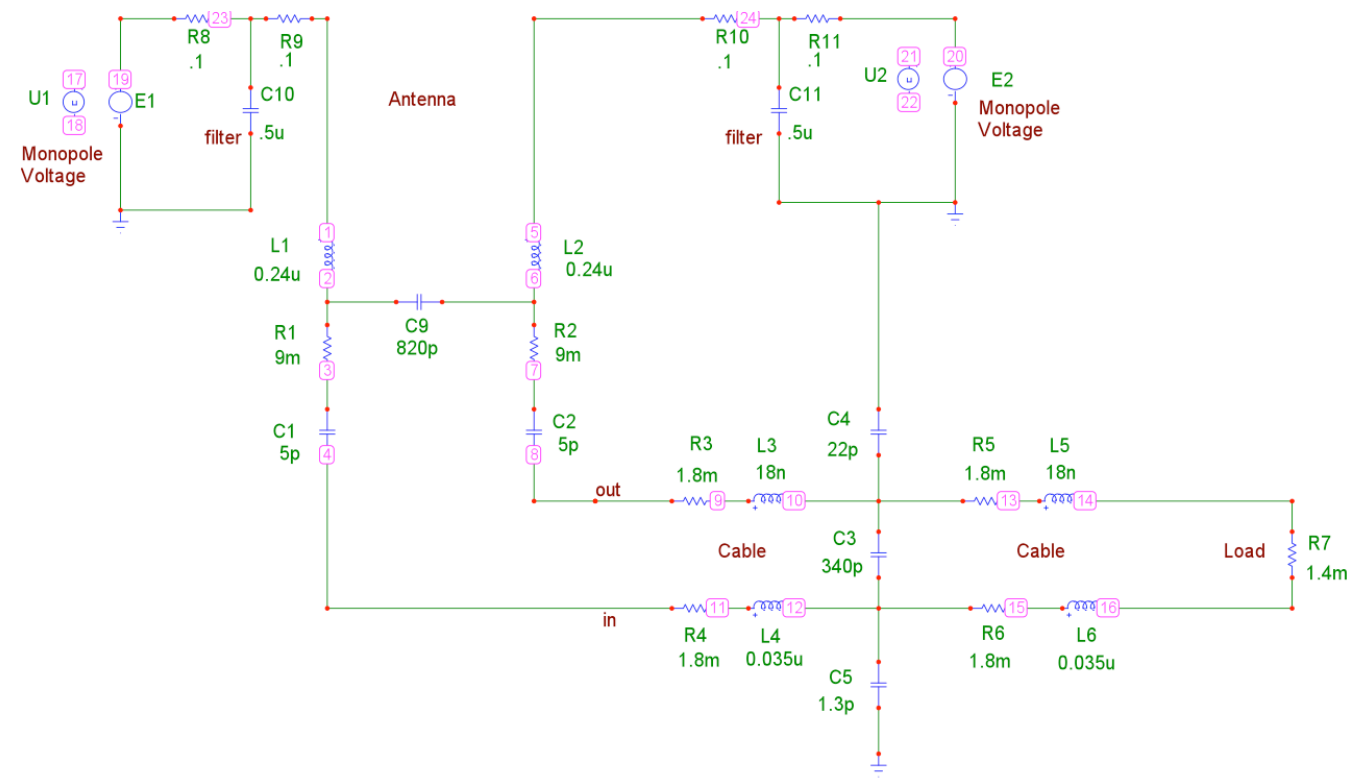

Figure 3.5.1. Representative circuit model for computing loaded antenna voltage.

Comparisons of the voltages calculated from the equations and EM simulations for the various antenna configurations are given in Table 3.1. These dipoles were not connected to a load, and the statements are valid for parameter ranges listed in Figure 3.1. Loading effects will be discussed in the next section dealing with laboratory measurements. 


\begin{tabular}{|c|c|c|l|}
\hline Dipole Type & Application & $\begin{array}{c}\text { Simulation vs. } \\
\text { Equation Volt }\end{array}$ & \multicolumn{1}{|c|}{ Notes } \\
\hline Thin & $\begin{array}{c}\text { Conventional } \\
\text { antenna }\end{array}$ & Good agreement & Eq. error $<<10 \%$ \\
\hline Fat & $\begin{array}{c}\text { Drum radius }> \\
\text { total length/17 }\end{array}$ & $\begin{array}{c}\text { Fair agreement } \\
\text { Equation higher }\end{array}$ & $\begin{array}{l}\text { Eq. error }<\mathrm{x} 2 \\
\text { Eq. conservative }\end{array}$ \\
\hline Unbalanced & $\begin{array}{c}\text { Cable length } \neq \\
\text { Drum length }\end{array}$ & $\begin{array}{c}\text { Good agreement } \\
\text { Equation higher }\end{array}$ & $\begin{array}{l}\text { Small effect } \\
\text { Eq. error }<10 \%,<1: 10 \text { ratio } \\
\text { Eq. conservative }\end{array}$ \\
\hline Gap in & $\begin{array}{c}\text { Gaps between } \\
\text { metal parts }\end{array}$ & Good agreement & $\begin{array}{l}\text { Minimal effect for practical small gaps } \\
\text { Eq. error }<10 \% \\
\text { Eq. conservative }\end{array}$ \\
\hline Grounded & $\begin{array}{c}\text { Grounded work } \\
\text { stations }\end{array}$ & $\begin{array}{c}\text { Poor agreement } \\
\text { Equation lower }\end{array}$ & $\begin{array}{l}\text { Modest effect } \\
\text { Eq. error } \approx 50 \% \\
\text { Eq. not conservative }\end{array}$ \\
\hline
\end{tabular}

Table 3.1. Comparison of dipole voltages from antenna equations and computer simulations.

The standard thin dipole equation is very accurate based on comparisons with the EM computer simulation away from the ground plane. For a given electric field, the antenna equation accurately calculates the open-circuit antenna voltage. The open-voltage level was checked against an electro-static simulation.

The fat-dipole equation does not apply to objects of interest in our safety analyses. The formula considers antenna radii up to one seventeenth of the total antenna length as fat. Applying the fat-dipole equation or even the thin dipole formula to a truly wide dipole arm, i.e., drum-shaped object, will produce voltages that are higher than indicated by the 3D EM model. Therefore, the equations are conservative. The difference between equation and simulation was less than a factor of 2 .

The unbalanced dipole voltage can be computed using the monopole equation. For armlength ratios of less than 10 , the effect mutual coupling effect between the arms is not significant, approximately $10 \%$. In realistic situations, there is good agreement between the equation and electro-static EM simulation. The equations produce conservative results.

For small gap the space between the arms has little effect on the dipole voltage, and the open-circuit voltage can be calculated with the antenna equation with good accuracy. The voltage generated by a dipole with a very large gap, $>10 \%$ of the total length, can be calculated with a modified antenna equation, Eq. 3.6. There is good agreement between the equation and electro-static simulation. The equations produce conservative results.

Applying the standard formulas to a dipole with a grounded arm will produce nonconservative results. Simulations show that the grounded-arm voltage is about $50 \%$ higher than from a floating dipole. Because of the size of and the non-conservative differences, we rate the 
comparison as poor. The grounded-arm configuration represents the possibly of grounding the drum-like container and/or work stand. 


\section{Experimental Validation}

Because the dipole equations and computer simulations could be used for critical safety analysis, a laboratory study was attempted to validate the accuracy and limitations of the equations and modeling codes. Even on the faster rising edge, the lightning current changes relatively slowly from an RF perspective, around a microsecond. Therefore, the lightning wavelength is much longer than the dimensions of the dipoles. These low radio frequencies allow the use of a 2-meter high transverse electromagnetic (TEM) cell to create known and reasonably spatially-uniform electric fields to excite our dipoles [1.6 - Crawford]. The study was performed in the frequency domain from $10 \mathrm{kHz}$ to over $1 \mathrm{MHz}$ using a commercial signal generator that produces a few voltages. There are two advantages to performing frequency domain studies rather than using lightning-like pulses. First, the sine waveform generates more average power for a given measurement period, and thus will improve the signal-to-noise ratio. Second, the frequency response of the antenna system provides important insights about the coupling efficiency in the spectrum of the lightning current. While the antenna has a flat response over the lightning current spectrum, adding the load will change the circuit frequency response. It acts like a high-pass filter. This is easier to observe in the frequency domain than in the time domain.

At all frequencies, the dipole voltage will drop with any loading, such as from a cable and digitizer. To minimize the impact of the measurement instrumentation on the antenna voltage, a highimpedance probe was. A Tektronix P6247, a highimpedance active differential, voltage probe with low input capacitance $(<1 \mathrm{pF})$ that significantly reduces the loading was selected [4.1 - Tektronix]. (See Figure 4.1.)

A 12-inch dipole was fabricated on a thin circuit board, and the antenna arms were fabricated on opposite sides of the thin board. Based on computer simulations, the higher than air dielectric constant around the arms has little impact in the antenna

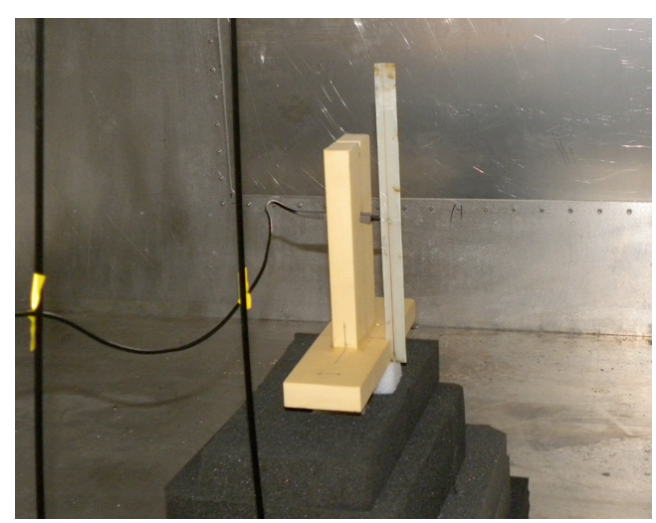

Figure 4.1. Baseline antenna used in validation study with differential highimpedance voltage probe. characteristics. Two small wires connecting the antenna to the probe were kept as short as possible, a few millimeters. A light-colored vertical foam board in the center of Figure 4.1 supports the probe. Near the dipole, the cable is routed on an equipotential line.

The connection diagram for the equipment used in the antenna characterization is shown in Figure 4.2. The output of the TEM cell was terminated in a $50 \Omega$ load to reduce reflections. 


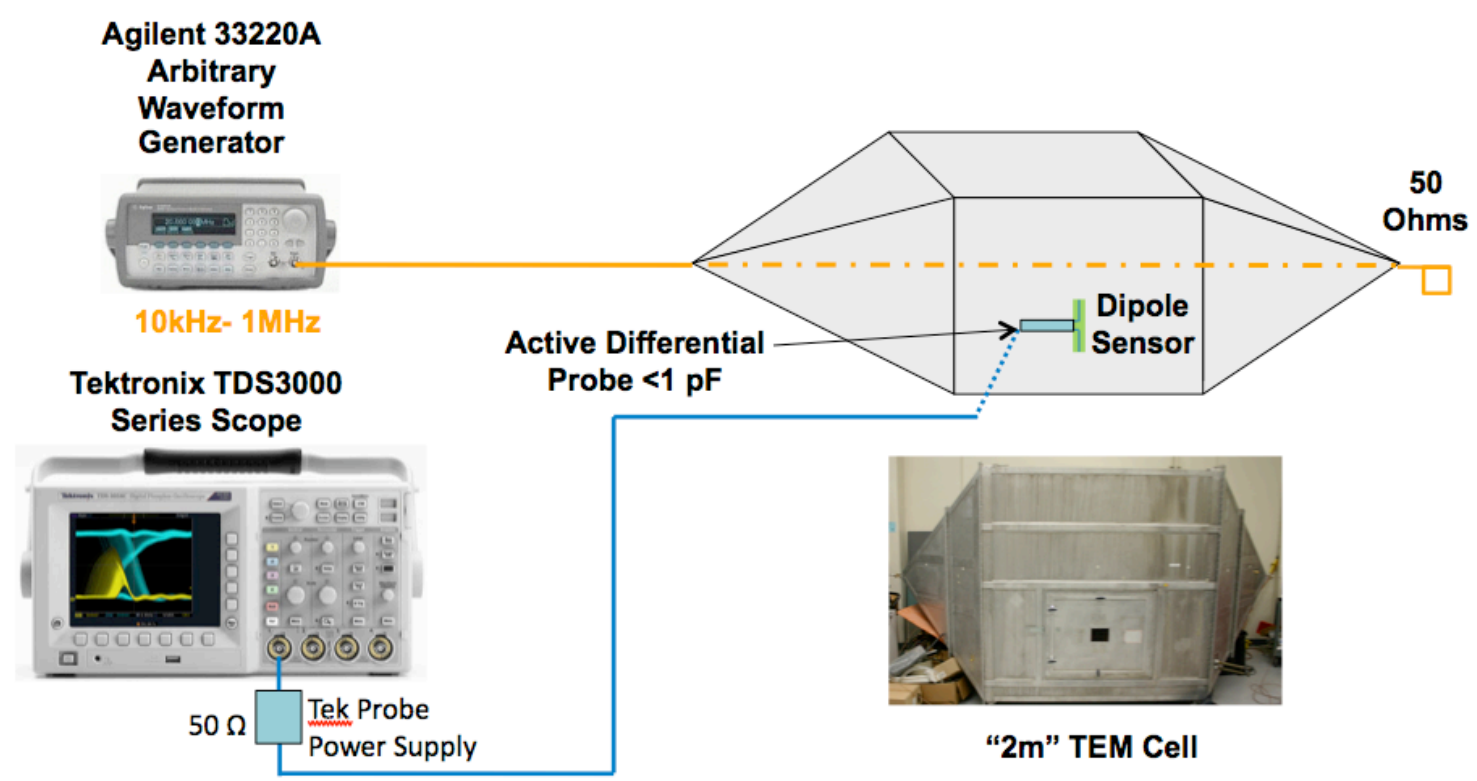

Figure 4.2. Connection diagram of equipment used in the antenna characterization study.

The fidelity of the cell was verified by comparing field measurements against the modeling results. A small, electrically isolated, commercial electric field probe (Lindgren $\mathrm{HI}-6105$ ) was used to measure the electric field [4.2 - Lindgren]. (See Figure 4.3.) The probe communicates with a computer through a fiber optic cable that minimizes distortion of the cell fields. This nonconductive cable also provides power to the field probe from a laser source.
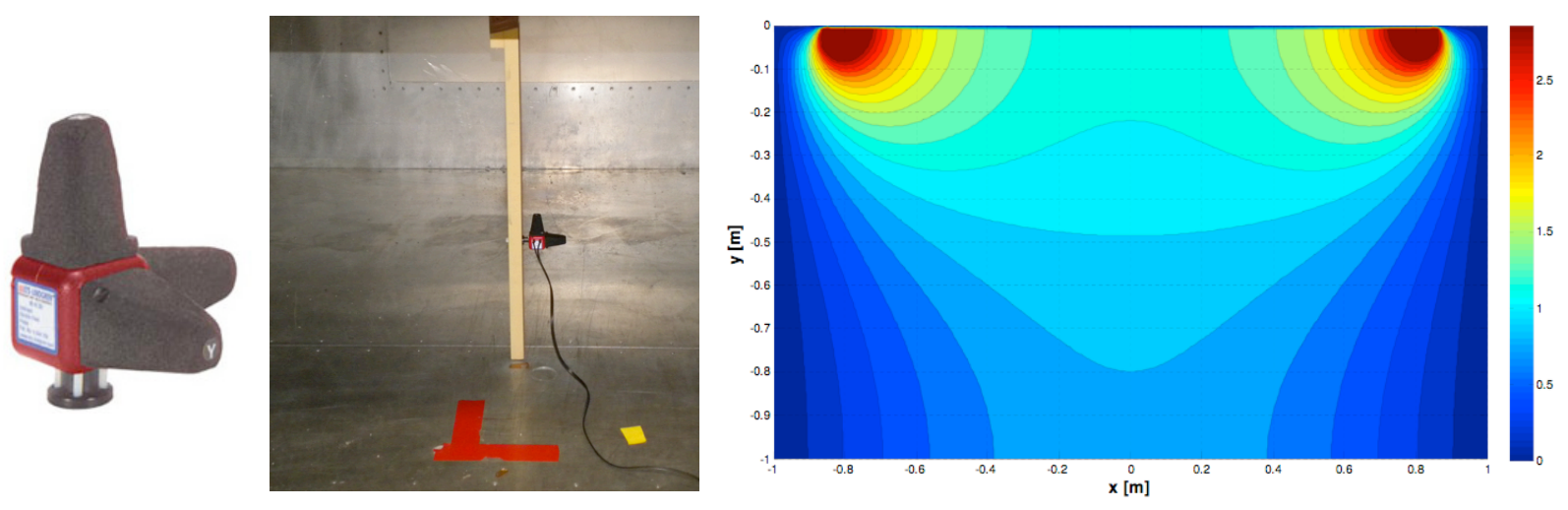

Figure 4.3. An electrically isolated E-field probe was used to check the TEM cell.

Support structures for the probe, antennas, and metallic cables were constructed from lowdensity foam that is mostly transparent to the RF field. For the antenna study, a digitizer using the averaging function measured the dipole voltages from the high-impedance probe. The digitizer was floated and operated on internal batteries to minimize the effect of a grounded instrumentation cable on the TEM cell fields. Where possible, the metallic instrumentation coaxial cable was routed along an equipotential line as shown in the plot provided by our 
colleague Adam White in Figure 4.4. The importance of the cable placement will be made clear in the fat antenna measurements.

\subsection{Measurement Results}

The measurement results will be presented in the same order followed in the simulation section: thin, fat, unbalanced, antenna gap, and grounded.

In order to compare the antenna 3D electro-static modeling results without a load against the measurements, a circuit

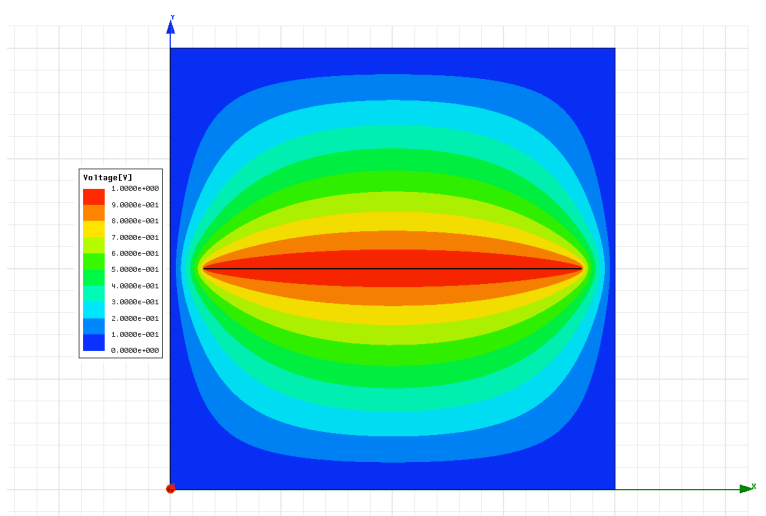

Figure 4.4. Equipotential lines in the TEM cell guide the routing of instrumentation cables. model that includes the loading from the probe was developed. In the circuit, the voltage source is determined from the electric field level in the TEM cell and the EM models.

\section{Thin Dipole}

The circuit diagram for the antenna and instrumentation is shown in Figure 4.1.1. The small capacitance values of the antenna, feed wires, and probe are very difficult to measure, and hence computed or specified quantities were used. The antenna capacitance of $1.05 \mathrm{pF}$ was computed for a 12-inch dipole with $1 \mathrm{~mm}$ radius, Eq. 3.5. The capacitance of the short twin-wire feed at the base of the antenna was also computed, Eq. 4.1. The $0.76 \mathrm{~mm}$-dia wires were separated by 3.1 $\mathrm{mm}$, and the length was estimated to be $3.3 \mathrm{~mm}$.

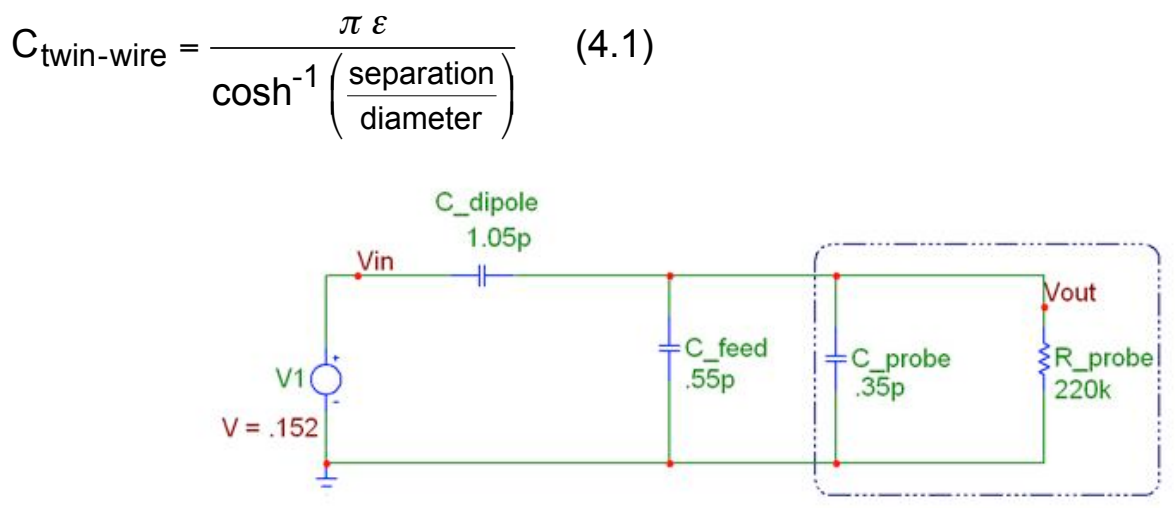

Figure 4.1.1. Circuit diagram of dipole, feed and high-impedance voltage probe.

The probe resistance was derived from the specifications for the probe; the published resistance of $200 \mathrm{k} \Omega$ was increased by $10 \%$ to better match the measured voltage. The probe capacitance, $0.35 \mathrm{pF}$, was inferred from the specification for the single-ended probe of $0.7 \mathrm{pF}$ added in series. The circuit diagram shows a required ground connection that was not present in the measurements. 
For the baseline dipole configuration with the antenna in the middle of the cell, there is very good agreement between the measured and theoretical voltage from $10 \mathrm{kHz}$ to $2 \mathrm{MHz}$. (See Figure 4.1.2.) Based on our understanding of the fields in the cell, the effect of routing the cable, and the accuracy of the probe and digitizer, we estimate our accuracy to be about $\pm 10 \%$ for the thin dipole measurement.

\section{Fat Dipole}

The fat dipole in the TEM cell is shown in Figure 4.1.3. The lower arm has a $6 "$ radius, and the fat antenna is elevated to the same position as the thin

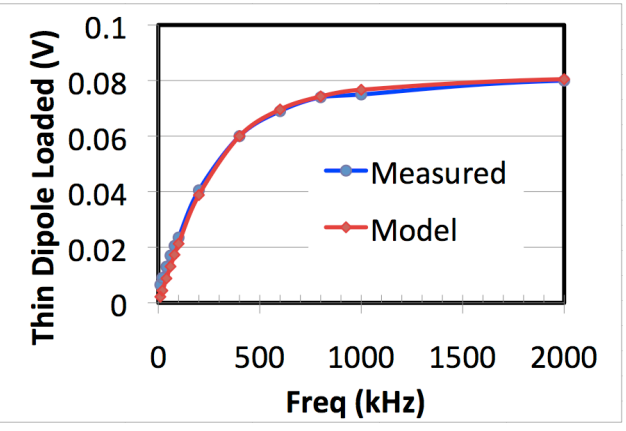

Figure 4.1.2. The measured voltages from a 12 " dipole match the modeling results. dipole. The thin upper element was constructed from $0.141 "$ solid-jacketed copper coaxial cable. The outer shield was stripped off, and the insulator was left in place to support and protect the thin inner wire. The coaxial cable from the high-impedance probe is supported with dielectric rods. In the figure, the cable can be seen lying on top of the fat lower arm.

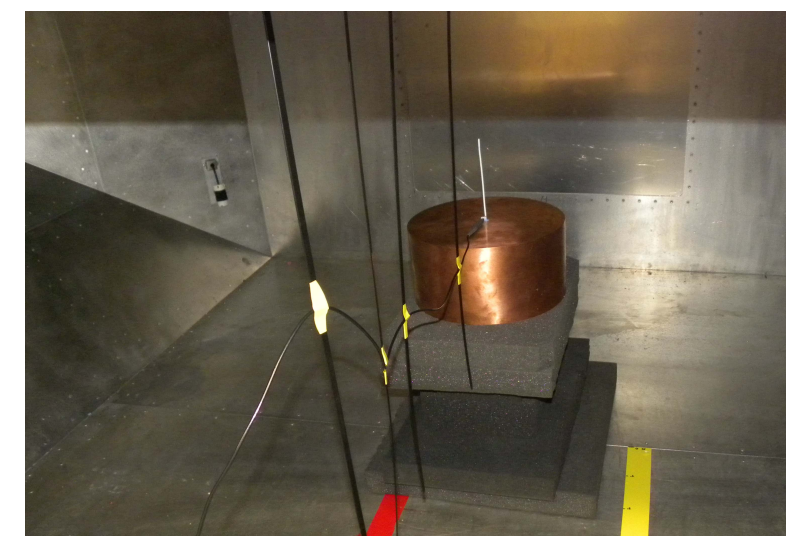

Figure 4.1.3. Fat dipole is 12 inch long, and the lower arm is 12 inches in diameter.

The circuit model for the fat dipole and load was modified with a larger antenna capacitance. (See Figure 4.1.4.) The $2.06 \mathrm{pF}$ value, $\mathrm{C} 1$, was determined from a 3D MAXWELL electro-static model of the fat dipole. The load components are unchanged.

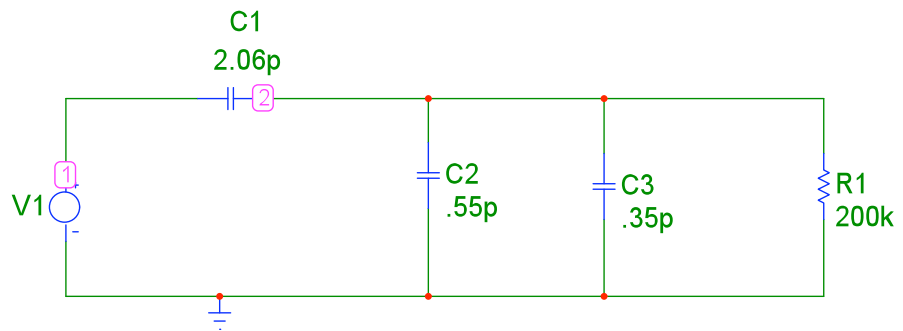

Figure 4.1.4. The fat-dipole circuit model had a larger antenna capacitance. 
Comparison of component values and voltages for the 12-inch thin and fat dipoles are shown in Table 4.1.1. The effective height multiplier for the fat dipole was obtained from the computer simulations. The effective height is the product of the multiplier times the antenna length. At the higher frequencies, the load factor depends on antenna capacitance divided by the sum of the antenna and load capacitances, see Eq. 2.7. When the fat dipole produces only $67 \%$ of the potential as the thin antenna (see Figure 3.1.2), the fat loaded voltage is reduced to only $83 \%$ thin voltage because of the larger fat antenna capacitance. The fat antenna has the capacity to deliver more current because of the lower impedance.

\begin{tabular}{|c|c|c|c|c|c|}
\hline Dipole Type & Arm Sizes & $\begin{array}{c}\text { Effective } \\
\text { Height Mult'r }\end{array}$ & $\begin{array}{c}\text { Dipole Voltage } \\
\text { for 1 V/m }\end{array}$ & $\begin{array}{c}\text { Circuit Load } \\
\text { Factor }\end{array}$ & $\begin{array}{c}\text { Voltage } \\
\text { Loaded }\end{array}$ \\
\hline Thin & $\begin{array}{c}6 \text { in x 1 mm-r } \\
6 \text { in x 1 mm-r }\end{array}$ & 0.483 & $0.153 \mathrm{~V}$ & 0.538 & $0.083 \mathrm{~V}$ \\
\hline Fat & $\begin{array}{c}6 \text { in x } 1 \mathrm{~mm}-\mathrm{r} \\
6 \text { in x 6 in-r }\end{array}$ & 0.321 & $0.098 \mathrm{~V}$ & 0.696 & $0.066 \mathrm{~V}$ \\
\hline
\end{tabular}

Table 4.1.1. The fat dipole generates less voltage than the thin dipole.

The predicted thin (shown green) and lower fat (blue) antenna voltages in the TEM cell with a $1 \mathrm{~V} / \mathrm{m}$ electric field are shown in Figure 4.1 .5 as a function of frequency.

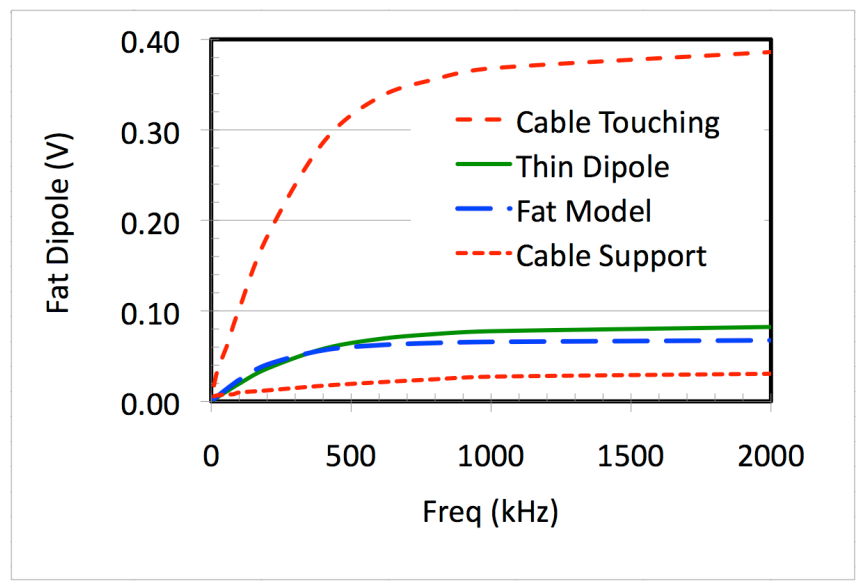

Figure 4.1.5. The fat-dipole voltages were difficult to measure.

The fat-dipole voltages were difficult to measure accurately, and they were either higher or lower than predicted, depending on the proximity of cable to the top surface of the fat arm. The discrepancy between predicted and measured voltages is caused by the potential generated by the cable in the TEM cell acting as a third arm in the antenna system. The electric fields and equipotential lines around the fat dipole are shown in Figure 4.1.6. If the cable does not follow an equipotential line, it will increase or decrease the dipole voltage through capacitive coupling from the shield of the cable and probe. 

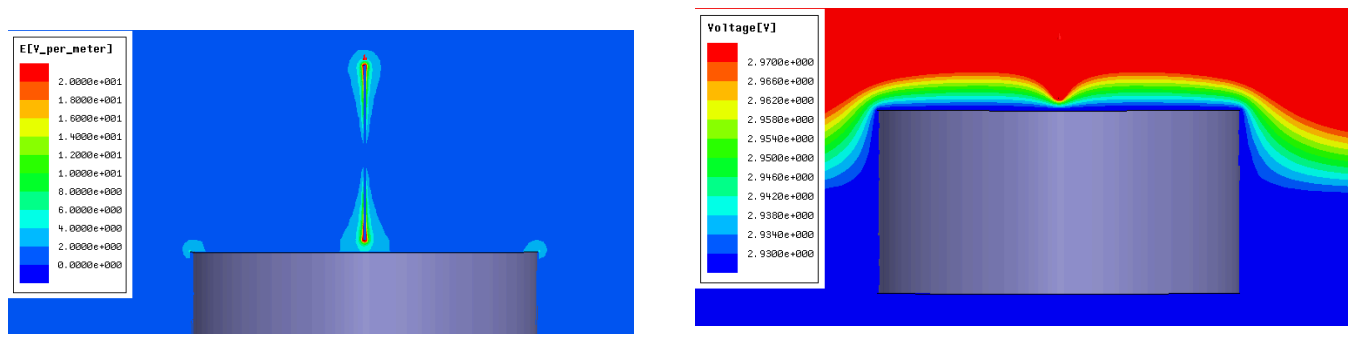

Figure 4.1.6. The electric fields and equipotential lines around the fat-dipole arm.

When the cable was touching the fat lower arm, as in Figure 4.1.3, the loaded antenna voltage was higher. When the cable was lifted off the lower arm, the voltage dropped. (See Figure 4.1.7.) The laboratory validation has uncertainty, but the predicted voltage was bracketed by the measurements. We believe that the EM field and circuit modeling is accurate, and it is the instrumentation that needs to be improved. One possible upgrade is to add an optical link between the high-impedance voltage probe and digitizer. This can be accomplished by putting a battery source, voltage-to-fiber-optic-cable converter and the probe in the base of the fat arm. The thin arm would pass through a hole in the fat element, and the measurement would be done inside the closed cylinder. The fat cylinder would have a second hole for the fiber optic cable.

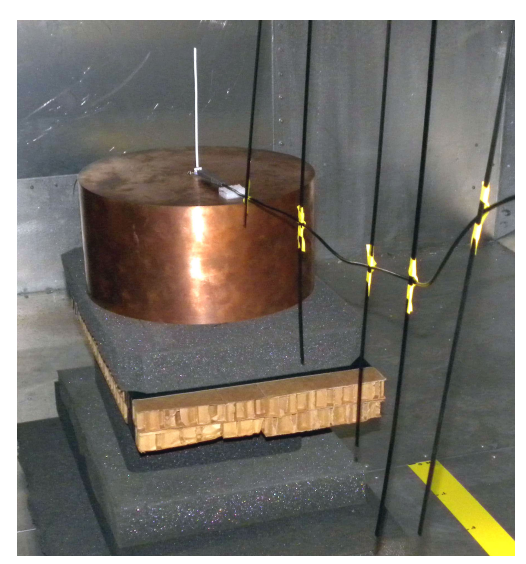

Figure 4.1.7. The fat-dipole voltage with cable elevated from the lower arm is smaller.

\section{Unbalanced Dipole}

For slightly unbalanced dipoles, the standard monopole antenna equation will produce reasonably accurate voltages. See plot in Figure 3.2.2. The mutual coupling (or "shielding") effect identified by the electro-static model is not included in the standard formula. The unbalanced dipole model was not checked in the TEM cell for a couple of reasons.

First, consider the configuration where the length of the shorter arm is 0.6 -inch, $10 \%$, of the 6 -inch arm. The voltage predicted by the monopole equation for the unbalanced dipole is reduced by $40 \%$ from the 12 -inch balanced antenna. The short $10 \%$ arm antenna capacitance is reduced by about four. The reduced load voltage would be difficult to measure accurately. A bigger concern is error introduced by the routing of the probe cable.

Second, the difference between the voltages calculated from the equation and from the EM model that includes the mutual coupling is about $12 \%$. (See Figure 3.2.2.) Our laboratory measurements are not accurate enough to confidently resolve the small difference created by the mutual "shielding". 
Instead of a measurement-based validation, a different analyst completed a second computer simulation using a different code described in Appendix A. The antenna voltages from the two simulations for the unbalanced dipole showed excellent agreement. The performance of unbalanced dipoles is predictable, much like the thin balanced dipoles. The standard monopole equation would produce conservative results.

\section{Antenna Base Gap}

The gap between the arms has two potential effects: voltage loading for very small separations and voltage increase for widely separated arms. Theoretically the second effect might be useful in a safety analysis. Practically, while the larger arm separation would produce a higher potential, the electric field in the gap will not increase. The voltage breakdown level also increases, and hence large gaps may not be a concern. Therefore, the laboratory validation incorporated a small gap in the dipole.

The thin dipole gap is about $3 \mathrm{~mm}$ for the validation. This small separation causes very little loading, and the predicted antenna voltage should be reduced by about $1 \%$. See plot in Figure 3.3.2. This small effect could not be measured given our accuracy of around $10 \%$. The laboratory measurement showed that the voltage reduction by the gap could not be large. This indirect validation confirms the lack of an effect with the $3 \mathrm{~mm}$ gap.

\section{Grounded Dipole}

In the modeling, the grounded dipole produced surprising results with the antenna voltage increasing by $50 \%$. The grounded lower arm of the dipole is shown in Figure 4.1.8. Measuring the voltage on the thin dipole was easier because the constant potential lines are less convoluted than around the fat antenna arrangement. To reduce the effect of the cable position changes on the dipole voltage, in the grounded-antenna measurement, the cable movement was minimized. The floated dipole was elevated only $1 \mathrm{~cm}$ from the bottom of the TEM cell. The predicted antenna voltage generated at $1-\mathrm{cm}$ from the floor should be only $2 \%$ higher than at the center of the cell. (See plot in Figure 3.4.2.)

The measured floating dipole voltage (green line) is shown in Figure 4.1.9. The predicted "grounded" voltage labeled

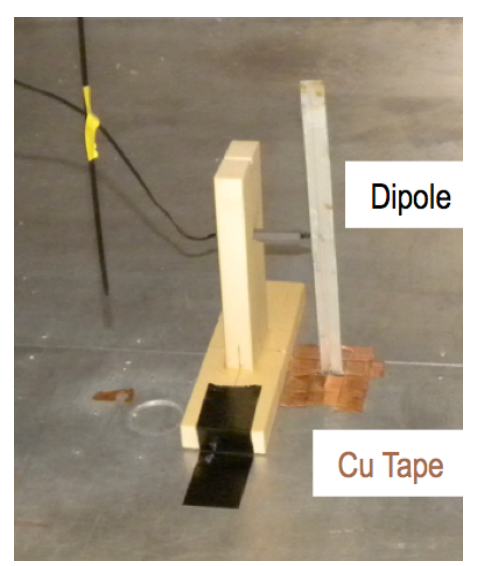

Figure 4.1.8. The lower tip of the dipole is connected to the TEM cell with copper tape. "From Model" (blue line) was calculated from the 1-cm above ground, floating, dipole voltage multiplied by 1.5. The measured grounded-dipole voltage (red line) is about $10 \%$ higher than predicted. Given the problem with cable routing and the low voltages, the accuracy is acceptable. 


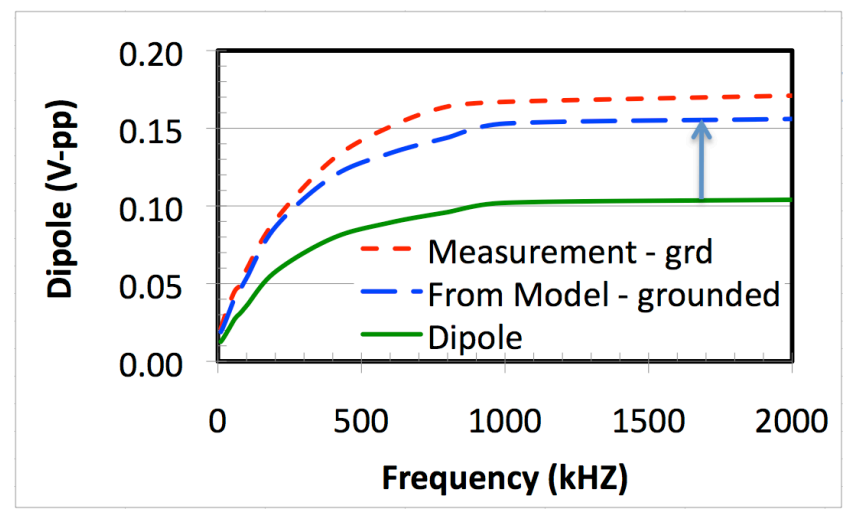

Figure 4.1.9. The dipole antenna touching ground plane produces approximate $50 \%$ more voltage than the floating dipole.

The laboratory validation of the computer models was successful for the thin dipole and the grounded dipole. The differences between the fat-dipole measurements and simulation results were large, and the discrepancies were explained. The fat-dipole effect is small when comparing load voltage against an equivalent-height conventional thin dipole. The gap between the antenna arms was about $3 \mathrm{~mm}$ and should have minimal effect on the induced voltage. This was validated in the thin dipole measurements that agreed well with the simulation and the equations. 


\section{Conclusions and Summary}

The two goals of the unconventional dipole study were (1) to understand the limitations of standard antenna equations developed for thin dipoles when applied to irregular antennas, and (2) to validate the RF-coupling modeling tools that would be used in indirect-lightning safety analysis. Computer modeling is the main tool for the safety analysis, and therefore the software validation is important.

The validation process consists of comparing voltages from antenna equations, computer models and laboratory measurements. There were differences between the analytical results and computer simulations. The discrepancies were often small, $10 \%$; and were less than a factor of two. The laboratory study was designed to check the important configurations - grounded and fat dipoles. The laboratory portion of the validation was mostly successful. There were difficulties in measuring the fat-dipole voltage. The measurement instrumentation interacted with the fields and could be improved. A summary of the comparisons is listed in Table 5.1.

\begin{tabular}{|c|c|c|c|c|}
\hline Dipole Type & Application & $\begin{array}{l}\text { Simulation vs. } \\
\text { Equation Volt }\end{array}$ & $\begin{array}{l}\text { Simulation vs. } \\
\text { Measured Volt }\end{array}$ & Notes \\
\hline Thin & $\begin{array}{l}\text { Conventional } \\
\text { antenna }\end{array}$ & Good agreement & Good agreement & $\begin{array}{l}\text { Simulation error }<<10 \% \\
\text { Eq. error }<<10 \%\end{array}$ \\
\hline Fat & $\begin{array}{l}\text { Drum radius }> \\
\text { total length } / 17\end{array}$ & $\begin{array}{l}\text { Fair agreement } \\
\text { Equation higher }\end{array}$ & $\begin{array}{l}\text { Poor agreement } \\
\text { Bracketed by } \\
\text { measurements }\end{array}$ & $\begin{array}{l}\text { Small effect when loaded } \\
\text { Simulation error }<10 \% \\
\text { Eq. conservative }\end{array}$ \\
\hline Unbalanced & $\begin{array}{l}\text { Cable length } \neq \\
\text { Drum length }\end{array}$ & $\begin{array}{l}\text { Fair agreement } \\
\text { Equation higher }\end{array}$ & $\begin{array}{l}\text { No measurements } \\
\text { 3D simulation vs. } \\
\text { 2D simulation }\end{array}$ & $\begin{array}{l}\text { Small effect } \\
\text { Simulation error }<10 \% \\
\text { Eq. conservative }\end{array}$ \\
\hline $\begin{array}{c}\text { Gap in } \\
\text { antenna base }\end{array}$ & $\begin{array}{l}\text { Gaps between } \\
\text { metal parts }\end{array}$ & Good agreement & Good agreement & $\begin{array}{l}\text { Minimal effect } \\
\text { Simulation error }<10 \% \\
\text { Eq. conservative }\end{array}$ \\
\hline Grounded & $\begin{array}{c}\text { Grounded work } \\
\text { stations }\end{array}$ & $\begin{array}{l}\text { Poor agreement } \\
\text { Equation lower }\end{array}$ & Good agreement & $\begin{array}{l}\text { Modest effect } \\
\text { Simulation error }<10 \% \\
\text { Eq. not conservative }\end{array}$ \\
\hline
\end{tabular}

Table 5.1. Comparison of dipole voltages from antenna equations, computer simulations, and laboratory measurements.

The standard thin dipole equation, simulations and laboratory measurement produce very consistent voltages that including loading. A circuit model was used to evaluate the effect of antenna voltage loading in the validation process.

The laboratory validation of the voltages produced by fat-dipole equation and simulation was difficult. The uncertainty in the measurement was higher than desired. When loading was 
included in the voltage calculation, the effect of the fat versus thin dipoles was reduced. The loaded 12-inch fat dipole should have produced slightly less voltage than the thin dipole loaded with the high-impedance probe. There are good reasons to believe that the calculated load voltages derived from the antenna equation or electro-static simulation and the circuit model are accurate. The error in the analysis should be less than a factor of two.

For an unbalanced dipole with an arm-length ratio of less than 10, the mutual coupling effect on the voltage between the arms is not significant, $\leq 12 \%$. Because of the measurement uncertainties, no laboratory check was performed. The 3D simulation was validated with a different 2D simulation code, and there was also good agreement with the monopole equation.

The unbalanced dipole voltage can be computed using the monopole equation. For armlength ratios of less than 10 , the mutual coupling effect between the arms is not significant, $\leq$ $12 \%$. Because of the measurement uncertainties, no laboratory check was performed. The 3D simulation was validated with a different 2D simulation code.

The gap between the dipole arms had little effect on the voltage. Voltage measurement of the 12 -inch thin dipole with a $3 \mathrm{~mm}$ gap showed no effect. Practically, the antenna arm gap does not significantly affect the voltage.

For a dipole with a grounded arm, simulations and measurements show that the antenna voltage is about $50 \%$ higher than from a floating dipole. The difference between the simulated and measured voltage was about $10 \%$. The dipole equation gives non-conservative voltages, and the error is less than a factor of two. For the indirect-lightning threat, it is better not to ground the container or work stand.

From the comparisons of antenna equations, computer simulations, and laboratory measurements, we believe that the computer simulation of indirect-lightning RF coupling is valid. The standard dipole equations are sufficiently accurate for estimating antenna voltages with an understanding that the uncertainty could be as high as a factor of two. Electro-static and circuit models will compute detonator voltages more accurately. (See Table 5.2.)

\begin{tabular}{|l|c|c|c|}
\hline & $\begin{array}{c}\text { Dipole } \\
\text { Formula }\end{array}$ & $\begin{array}{c}\text { Dipole } \\
\text { EM Model }\end{array}$ & $\begin{array}{c}\text { Load } \\
\text { Circuit Model }\end{array}$ \\
\hline Uncertainty & $\mathrm{x} 2$ & $\pm 10 \%$ & $\pm 10 \%$ \\
\hline Effect & mostly conservative & $\begin{array}{c}\text { better for } \\
\text { unconventional } \\
\text { antennas }\end{array}$ & $\begin{array}{c}\text { reduce voltage } \\
\approx 1 / 2 \text { to } 1 / 100\end{array}$ \\
\hline
\end{tabular}

Table 5.2. Summary dipole formulas, and EM and circuit model accuracies. 


\section{Acknowledgement}

We thank Matt Suda and especially Homer Aycardo for making carefully measurements in the TEM cell. Homer also took the informative photographs. We appreciate the insights gained from Adam White's simulation and characterization of the TEM cell fields. We want to acknowledge Drs. Ron Streit and Constantine Hrousis for funding the work. We wish to talk Carol Richardson for proofreading and editing the report making it more readable.

\section{References}

[1.1] Clancy, T. J., C.G. Brown, M.M. Ong, G.A. Clark, "Lightning Protection Certification for High Explosive Facilities at Lawrence Livermore National Laboratory", 2006 IEEE Antenna and Propagation Society Conference, Albuquerque, NM.

[1.2] Brown Jr., Charles G., et al., "Numerical Calculation of the Spectrum of the Severe (1\%) Lightning Current and Its First Derivative", Feb 2010, LLNL-TR-423690, Lawrence Livermore National Laboratory.

[1.3] Tully, L. K., M. M. Ong, "Arc Energy Estimates: Applications in Lightning-Induced Concrete Spall", June, 2008, LLNL-TR-404460, Lawrence Livermore National Labortory.

[1.4] Schmitt, H.J., C.W. Harrison, Jr., C.S. Williams, Jr., "Calculated and Experimental Response of Thin Cylindrical Antennas to Pulse Excitation", Mar 1966, IEEE Transactions On Antennas and Propagation, pp 120-7.

[1.5] Crull, Eric W., Charles G. Brown, Jr., Mike P Perkin and Mike M. Ong, "Experimental Validation of Lightning-Induced Electromagnetic (Indirect) Coupling to Short Monopole Antennas", Aug 2008, LLNL-TR-405954, Lawrence Livermore National Laboratory.

[1.6] Crawford, Myron L., "Generation of Standard EM Fields Using TEM Transmission Cells", IEEE Transactions on Electromagnetic Compatibility, Vol. MC-16, No. 4, Nov 1974, pp 189 95.

[2.1] Kraus, John D, "Antenna", 1988, 2nd Ed, p. 41, McGraw-Hill.

[2.2] Schmitt, H. J., C. W. Harrison, C. S. Williams, Jr., "Calculated and Experimental Response of Thin Cylindrical Antennas to Pulsed Excitation", IEEE Transactions on Antenna and Propagation, vol AP-14, No. 2, March 1966, pp 120-7.

[2.3] Stutzman, Warren L., Gary A. Thiele, "Antenna Theory and Design", 2nd edition, 1998, pp 45-7, Wiley.

[2.4] Inan, Umran S., Aziz S Ina, "Engineering Electromagnetics", 1998, p 342, Addison Wesley. 
[3.1] Ansoft Corp., "MAXWELL 3D", v12.2, Aug 2010, http://www.ansoft.com/products/em/maxwell/

[4.1] Tektronix, "Differential Probes P6248 P6247 P6246", http://www2.tek.com/cmswpt/psdetails.lotr?ct=PS\&cs=psu\&ci=13502\&lc=EN

[4.2] Lindgren, "HI-6105 Electric Field Probe", http://www.ets-lindgren.com/page/?i=HI-6105 
Appendix A - High Resolution Models Of Unbalanced Dipoles - Javedani

Method 1: Zoom Technique in 2D MAXWELL
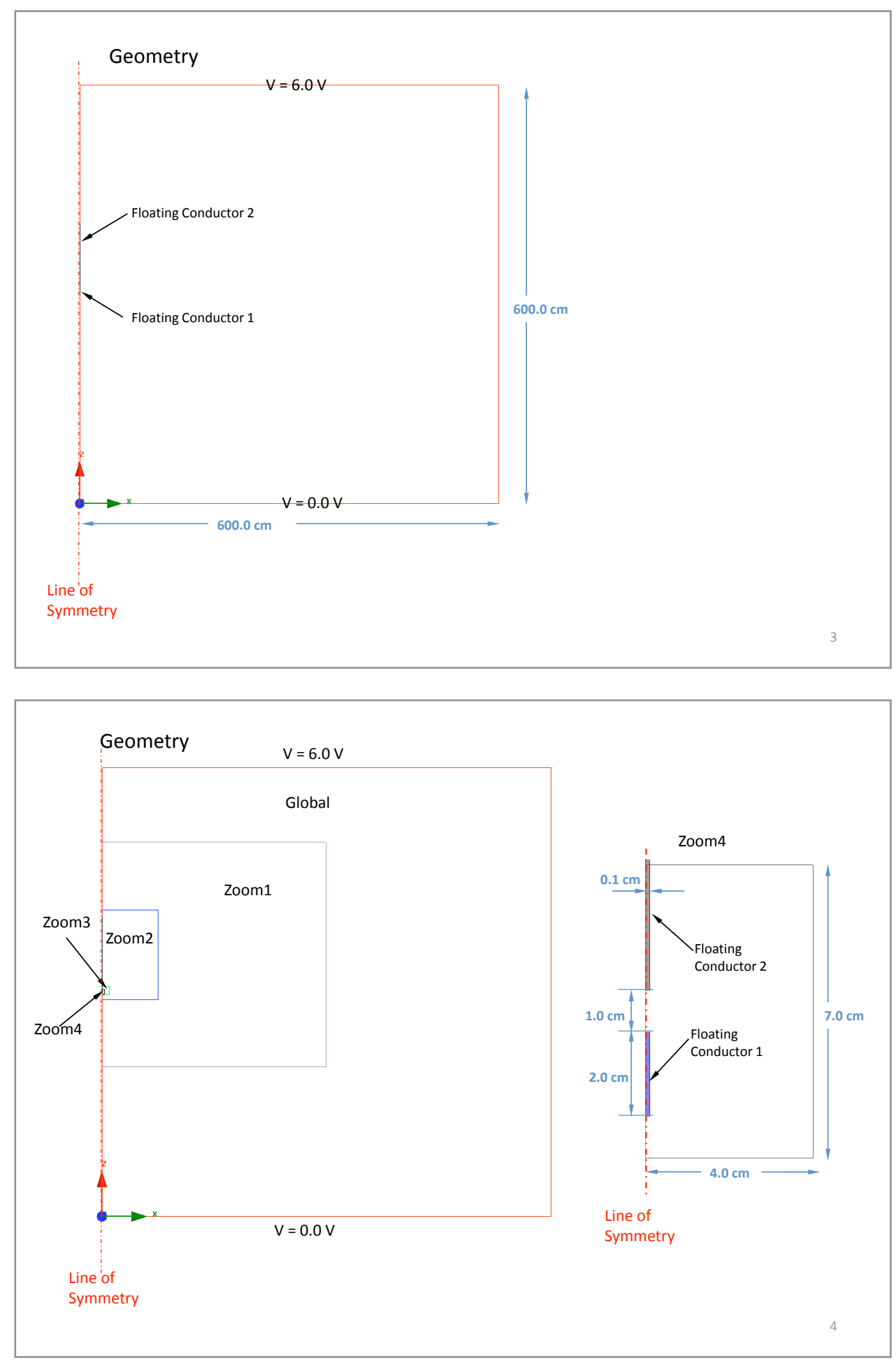
$\phi$ and $|E|$ in the rz plane for various zooms
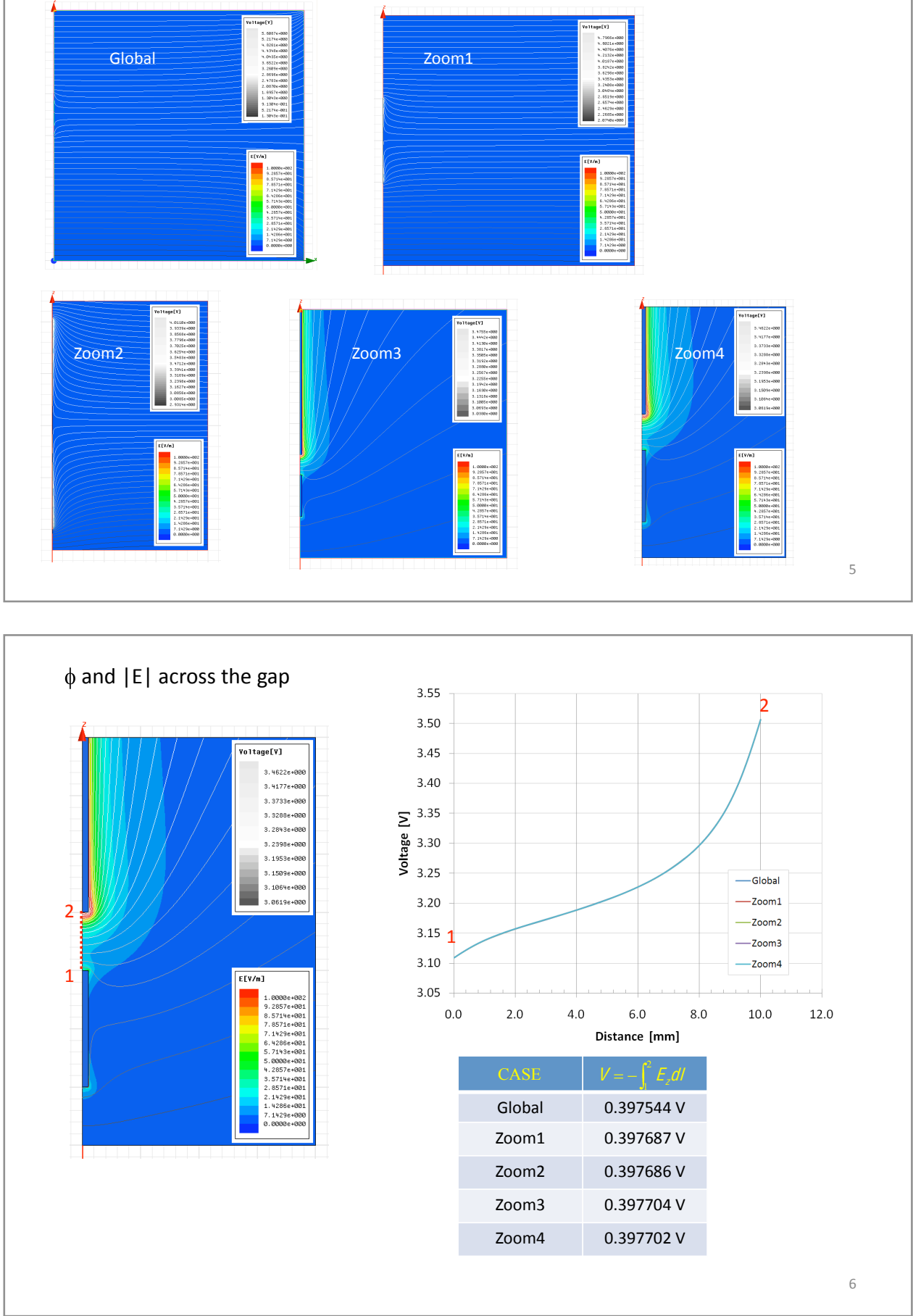


\section{Method 2: Dummy Geometry Technique in 2D}
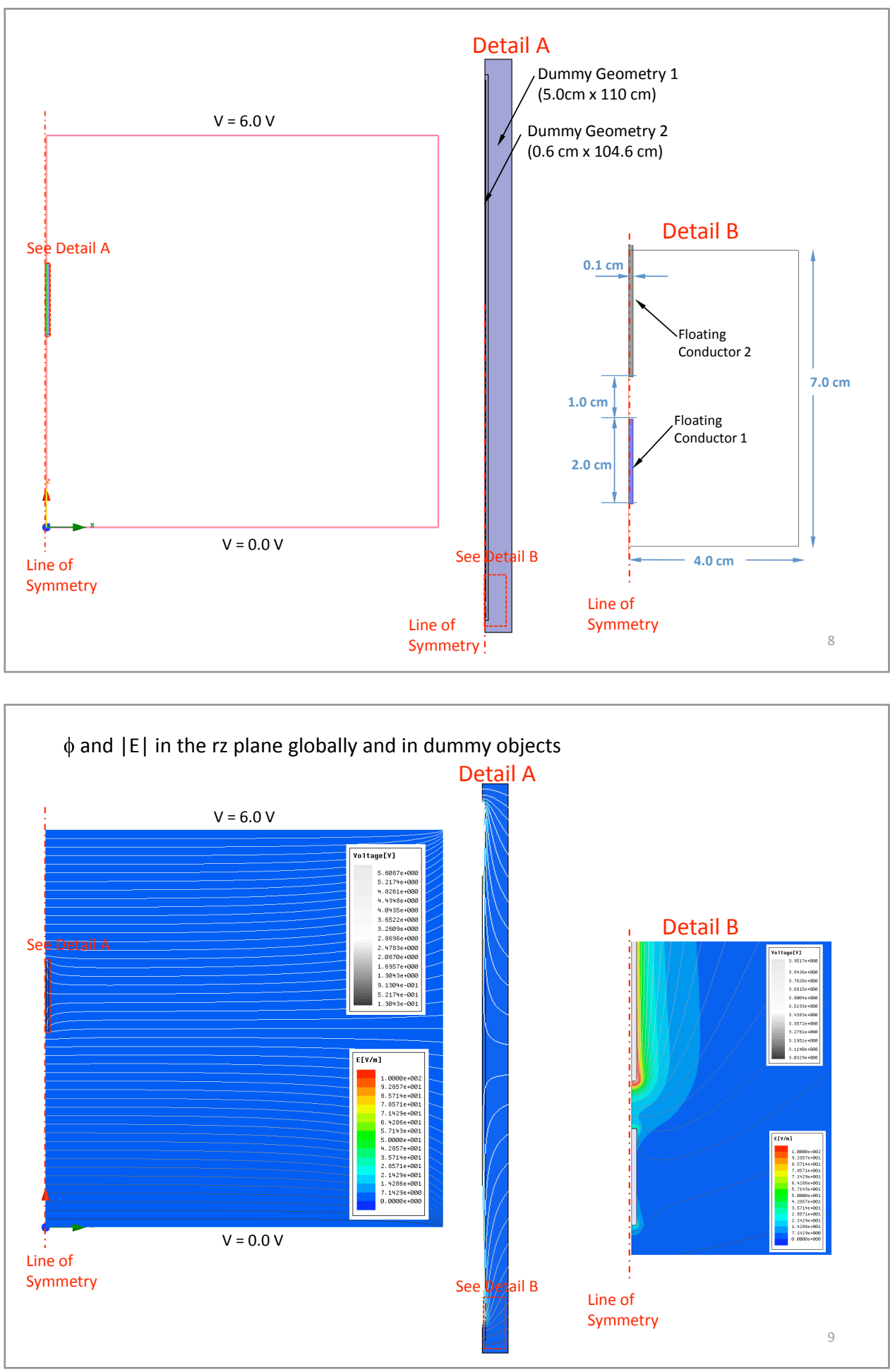


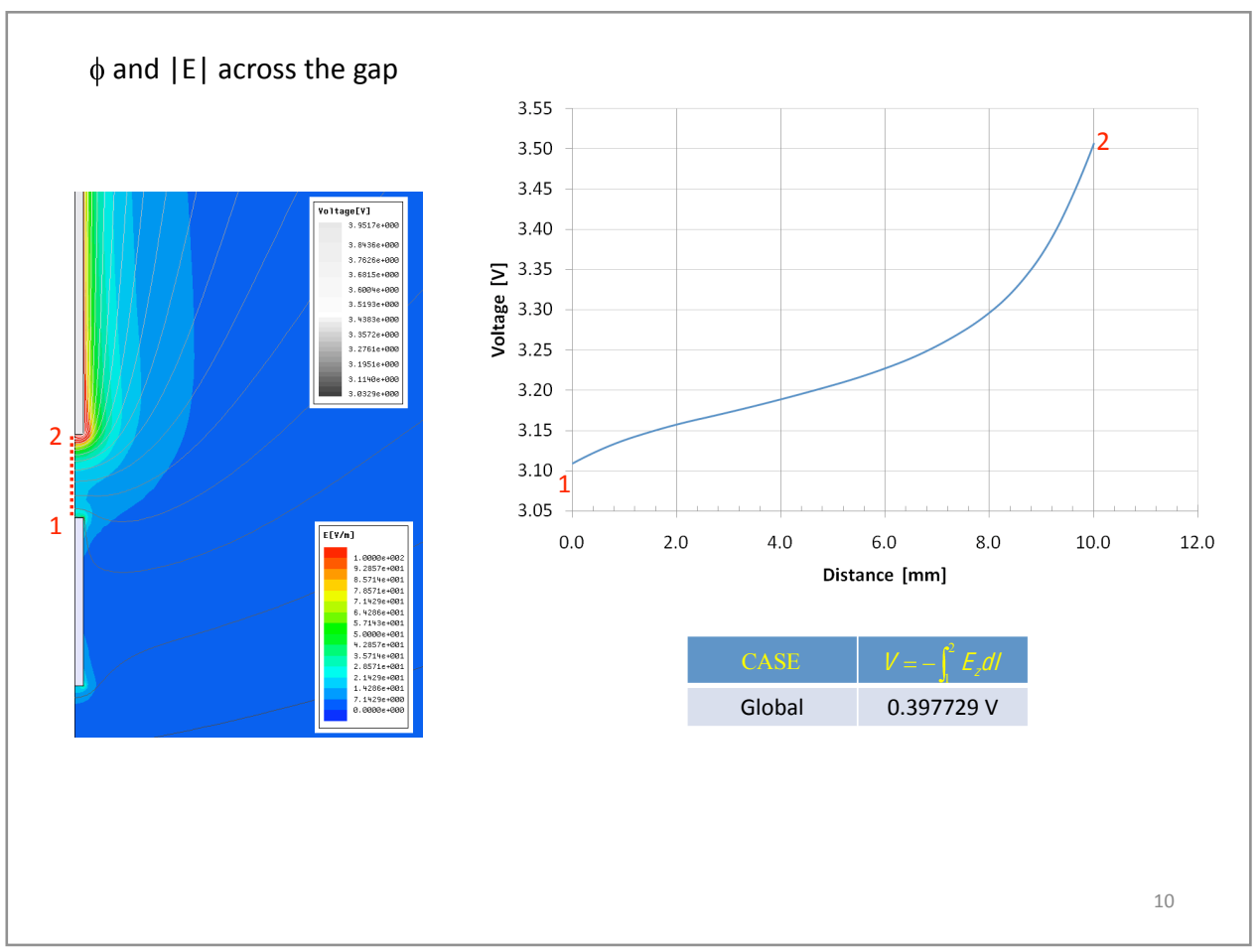

Method 3: Dummy Geometry Technique in 3D

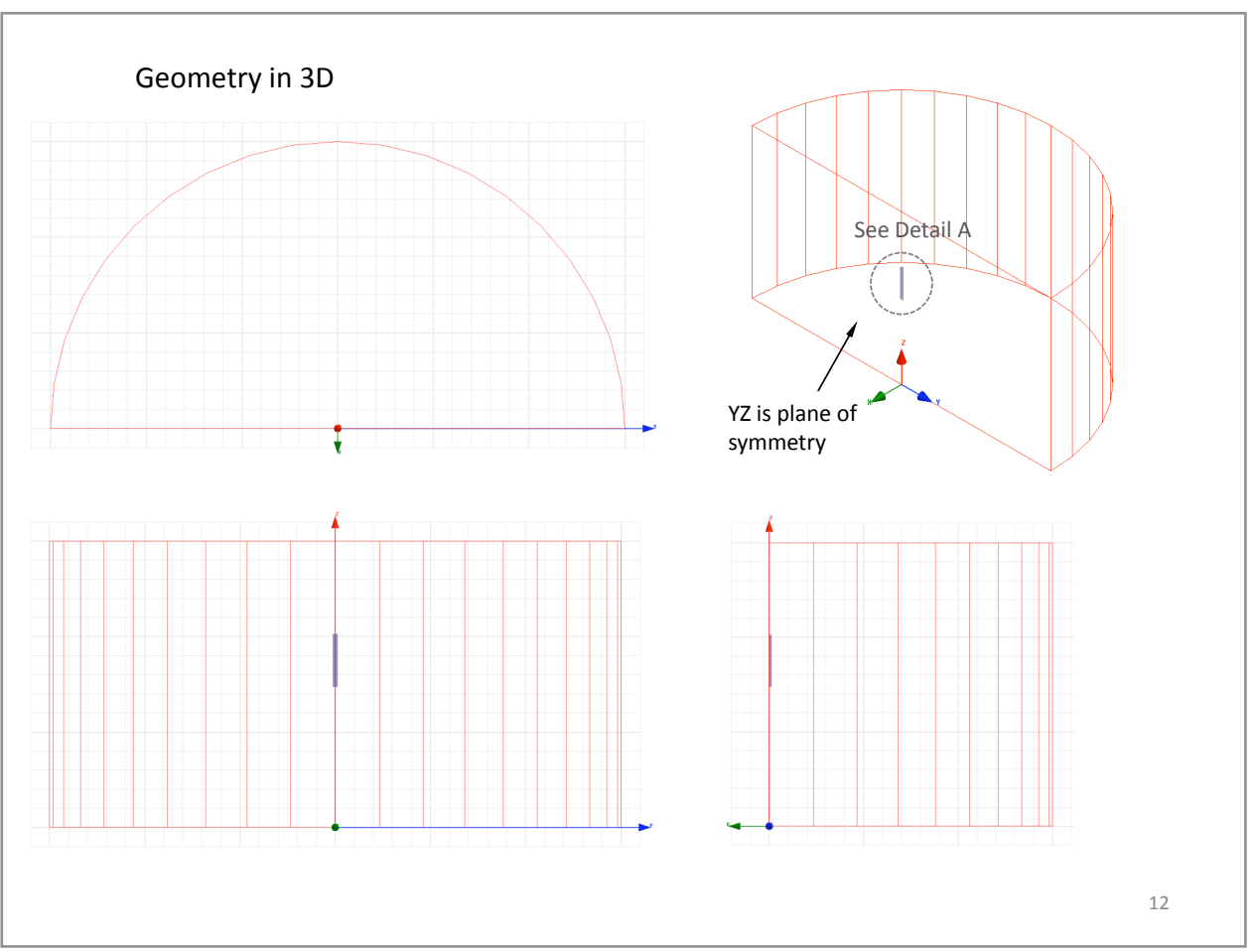



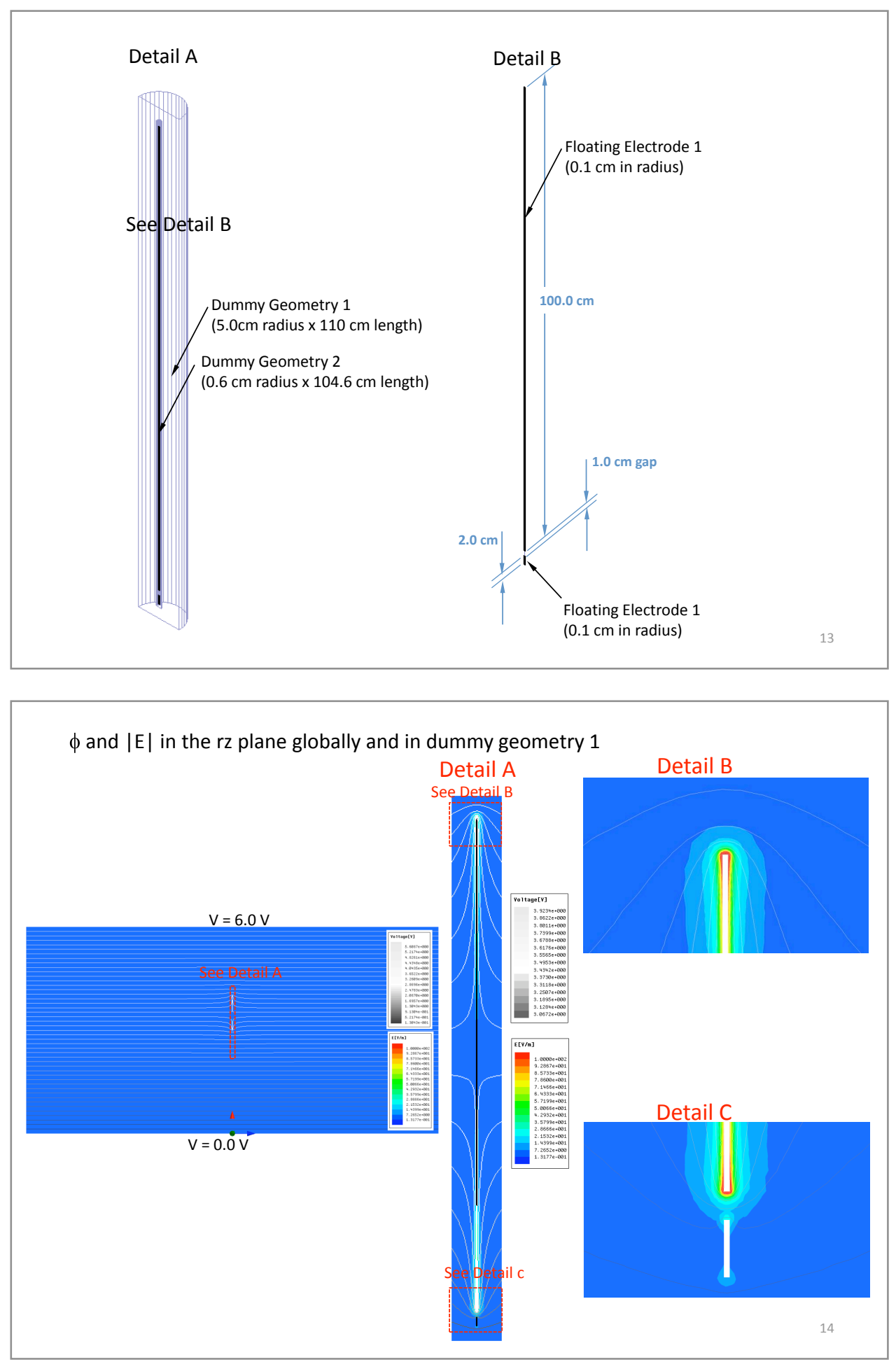


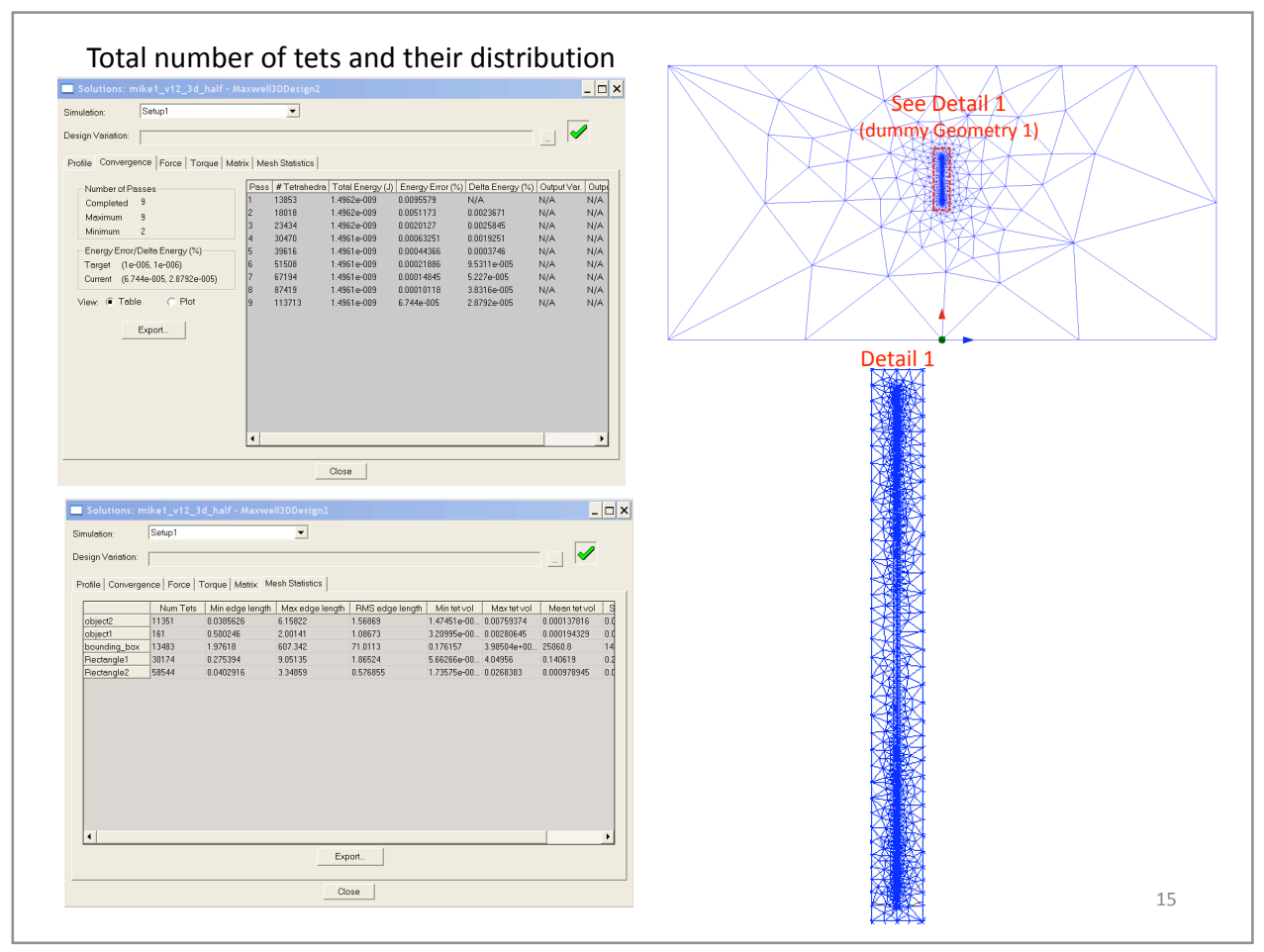

$\phi$ and $|E|$ across the gap

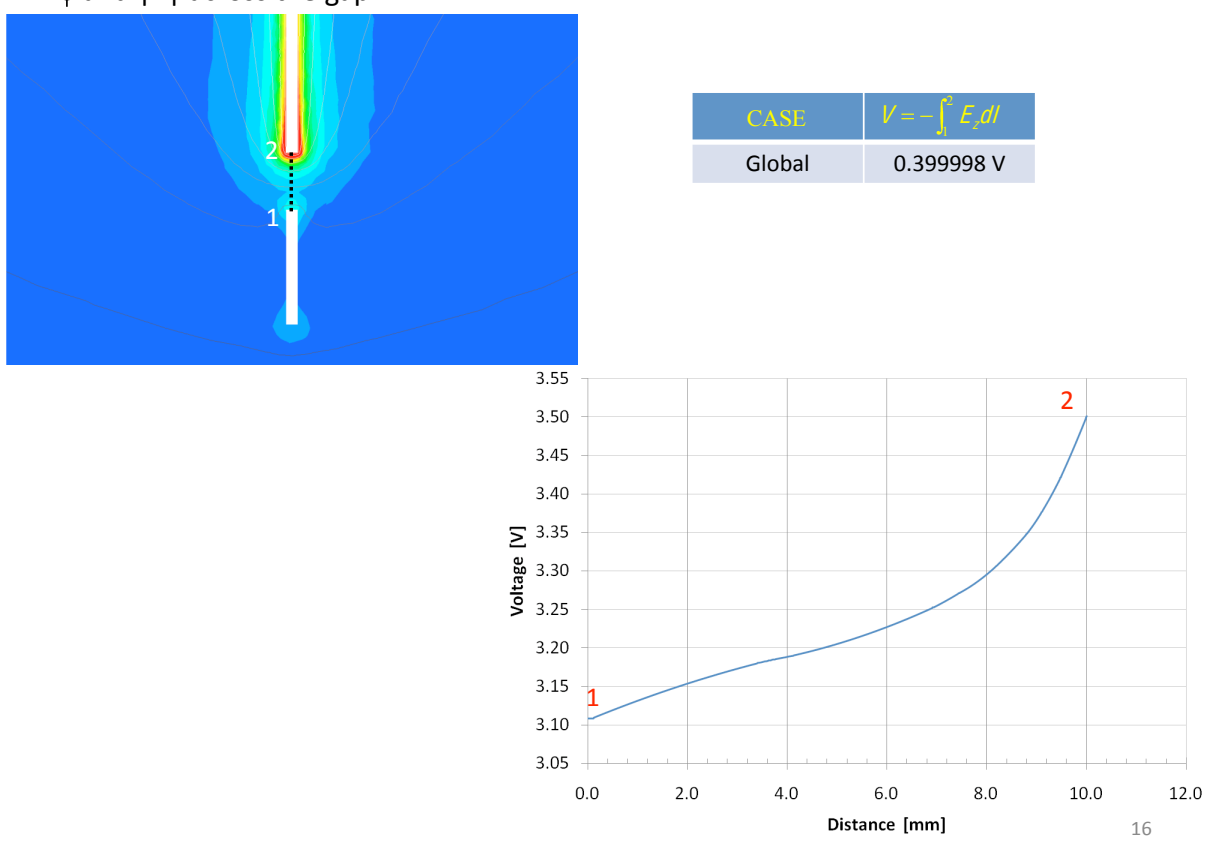




\section{Summary and Conclusions:}

Three different modeling methods were used to calculate the voltage across two floating conductors in a large solution space with Maxwell v12 (Electrostatic Solver).

Method 1 used 4 iterative zoom technique in 2D, where the $\phi$ value at the boundaries of each zoom $(n)$ was extracted from solving the previous zoom $(n-1)$ model.

The voltage across the gap for this case turned out to be $0.397702 \mathrm{~V}$. [See slide 6]

Method 2 used dummy geometries (2) in 2D to force the software to place the mesh in needed areas. This turned out to be the simplest method.

The voltage across the gap for this case turned out to be $0.397729 \mathrm{~V}$. [See slide 10]

Method 3 used dummy geometries (2) in 3D to force the software to place the mesh in needed areas. As many as $1.13 \mathrm{E}+05$ tets were created. Method 2 geometry was swept around

the z-axis (180 o) in order to generate the half-symmetry ( $r z$ plane) geometry.

The voltage across the gap for this case turned out to be $0.399998 \mathrm{~V}$. [See slide 16]

Surface Charges:

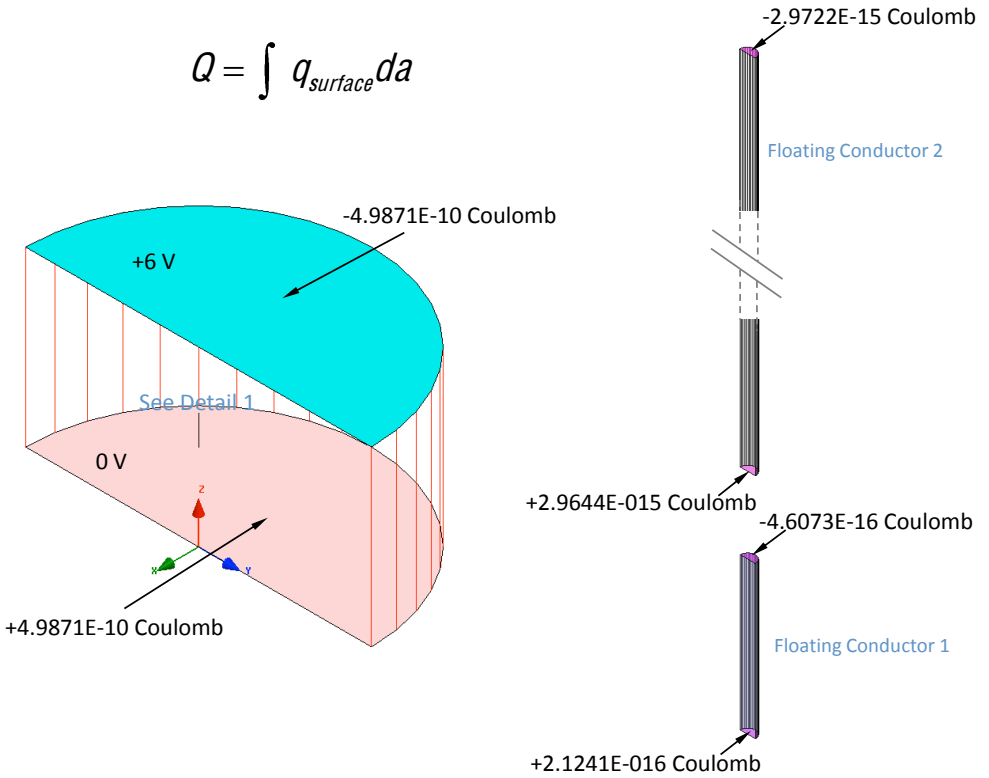

